BUTP-93/17

\title{
Perfect lattice action for asymptotically free theories⿴囗十
}

\author{
P. Hasenfratz and F. Niedermayerf \\ Institute for Theoretical Physics \\ University of Bern \\ Sidlerstrasse 5, CH-3012 Bern, Switzerland
}

July 1993

\begin{abstract}
There exist lattice actions which give cut-off independent physical predictions even on coarse grained lattices. Rotation symmetry is restored, the spectrum becomes exact and, in addition, the classical equations have scale invariant instanton solutions. This perfect action can be made short ranged. It can be determined by combining analytical calculations with numerical simulations on small lattices. We illustrate the method and the benefits on the $d=2$ non-linear $\sigma$-model.
\end{abstract}

\footnotetext{
${ }^{1}$ Work supported in part by Schweizerischer Nationalfonds

${ }^{2}$ on leave from the Institute for Theoretical Physics, Eötvös University, Budapest
} 


\section{Introduction and Summary}

In many theoretical problems in physics, at a given stage, numerical analysis is necessary. Numerical problems require some regularization, most often different meshes are used. If the lattice is coarse, the numerical procedure is relatively easy, but the results are contaminated by the artefacts of the regularization. It is a difficult problem to remove these artefacts by making the meshes systematically finer and then extrapolating.

We shall discuss the problem of artefacts in the context of lattice regularized asymptotically free quantum field theories. Removing the cut-off effects to reach the proper continuum limit is a central problem in this field [1]]. The method we describe, however, might find applications elsewhere, for example in solving partial differential equations.

In the study of quantum field theories cut-off effects show up in different ways. If the lattice is coarse grained then all the results are distorted, independently whether short or long distances are involved. Similarly, even on a fine lattice, at distances of a few lattice units the lattice structure, rather than physics dominates the results.

Removing the cut-off effects requires a careful analysis. Fig. 1 illustrates this process on the example of the running coupling in the $d=2$ non-linear $\sigma$-model [2]. The renormalized coupling at the distance scale $L$ is identified here with $m(L) L$, where $m(L)$ is the mass gap in a periodic box of size $L$. For small $L\left(L \ll 1 / m_{\infty}\right.$, where $m_{\infty}$ is the mass in the infinite volume) this quantity can be studied in perturbation theory and shows the properties of an asymptotically free coupling: as $L$ is changed, the coupling runs according to the perturbatively known beta-function. For larger values of $L$ the scale dependence of the coupling, for example the relation between $g(2 L) \equiv m(2 L) \cdot 2 L$ and $g(L) \equiv m(L) \cdot L$, requires a numerical calculation. A lattice is introduced with lattice unit $a$, and for a given $g(L)$ (in Fig. 1 $g(L)=1.0595) g(2 L)$ is calculated on lattices with $L / a=5,6,7, \ldots, 16$ and the results are extrapolated to $L / a=\infty$. This is a non-trivial numerical problem even in $d=2$.

A possibility to reduce lattice artefacts is to use improved actions. The idea behind this procedure is that for a large class of actions the physical predictions are universal while the cut-off effects are not. Although the history of improved actions is long [3, 4] they had a limited effect on actual calculations until now. The reason is that in some cases the proposed actions were derived using ad hoc assumptions, or uncontrolled approximations, in other cases they were fixed by perturbation theory and it is not clear whether in typical simulations they improve and to what extent. Deep in the continuum limit a perturbatively fixed action certainly improves. This is, however, not necessarily the case at moderate correlation lengths. Fig. 1 illustrates this problem also. Perturbation theory fails to reproduce 
even the sign of the observed cut-off effects [2].

A radical solution would be to use a perfect lattice action which is completely free of lattice artefacts. That such perfect actions exist follows from Wilson's renormalization group $(\mathrm{RG})$ theory [5, 6]. The aim of this paper is to show that in asymptotically free theories a combination of analytical and numerical techniques allows finding the perfect action to a sufficient precision. We consider an action perfect in practical calculations if even on a coarse grained lattice (correlation length $=\mathrm{O}(a))$, no cut-off effects can be seen in numerical simulations. The perfect action is not unique. This fact can be used to find a perfect action whose range of interaction is short and whose structure is relatively simple. Simulating such actions the gain/cost ratio can be very large.

In this work we study this problem in the $d=2, \mathrm{O}(3)$ non-linear $\sigma$-model, which we consider a pilot project, a preparation for $d=4$ Yang-Mills theories and QCD. A quadratic lattice is considered and the partition function is written as

$$
Z=\prod_{n} \int d \mathbf{S}_{n} \delta\left(\mathbf{S}_{n}^{2}-1\right) \mathrm{e}^{-\beta \mathcal{A}(\mathbf{S})}
$$

where $\beta \mathcal{A}(\mathbf{S})$ is some lattice representation of the continuum action

$$
\beta \mathcal{A}^{\text {cont }}(\mathbf{S})=\frac{\beta}{2} \int d^{2} x \partial_{\mu} \mathbf{S}(x) \partial_{\mu} \mathbf{S}(x)
$$

Beyond the basic requirements of $\mathrm{O}(3)$ symmetry, locality, correct classical limit, translation and $90^{\circ}$-rotation symmetry, the form of the lattice action is largely arbitrary. It might contain nearest neighbour, next-to-nearest neighbour, etc., even different multispin interactions. Let us denote the corresponding couplings by $c_{1}, c_{2}, \ldots$ The action $\beta \mathcal{A}(\mathbf{S})$ is represented by a point in the infinite dimensional space of couplings $\left(\beta, c_{1}, c_{2}, \ldots\right)$. We shall consider RG transformations in configuration space, namely block transformations with a scale factor of 2 . Under repeated block transformations the action moves in this coupling constant space. The expected flow diagram is sketched in Fig. 2 [6, 7]. In the $\beta=\infty$ hyperplane there is a fixed point $(\mathrm{FP}) c_{1}^{*}, c_{2}^{*}, \ldots$, whose exact position depends on the details of the block transformation. We shall use the notation $\mathcal{A}\left(\mathbf{S} ; c_{1}^{*}, c_{2}^{*}, \ldots\right)=\mathcal{A}^{*}(\mathbf{S})$ and call $\beta \mathcal{A}^{*}(\mathbf{S})$ the $\mathrm{FP}$-action. The $\mathrm{FP}$ has one marginal and infinitely many irrelevant directions. The marginal operator is $\mathcal{A}^{*}$ itself [6]. Actually, $\mathcal{A}^{*}$ is not exactly marginal, it is weakly relevant. If $\beta$ is very large, under a $\mathrm{RG}$ transformation with a scale factor 2 , $\beta \mathcal{A}^{*}(\mathbf{S}) \rightarrow \beta^{\prime} \mathcal{A}^{*}(\mathbf{S})$, where $\beta^{\prime}=\beta-\frac{1}{2 \pi} \ln 2$. The coupling $g=1 / \beta$ grows according to the beta-function of this asymptotically free theory. The trajectory which leaves the FP along the weakly relevant direction is called the renormalized trajectory (RT). For large $\beta$ the RT runs along the $\mathrm{FP}$-action, but for smaller $\beta$ they do not coincide anymore.

It is easy to see that the points of the RT define perfect actions. The argument goes as follows. At any given $\beta$, the point of the RT is connected to the infinitesimal 


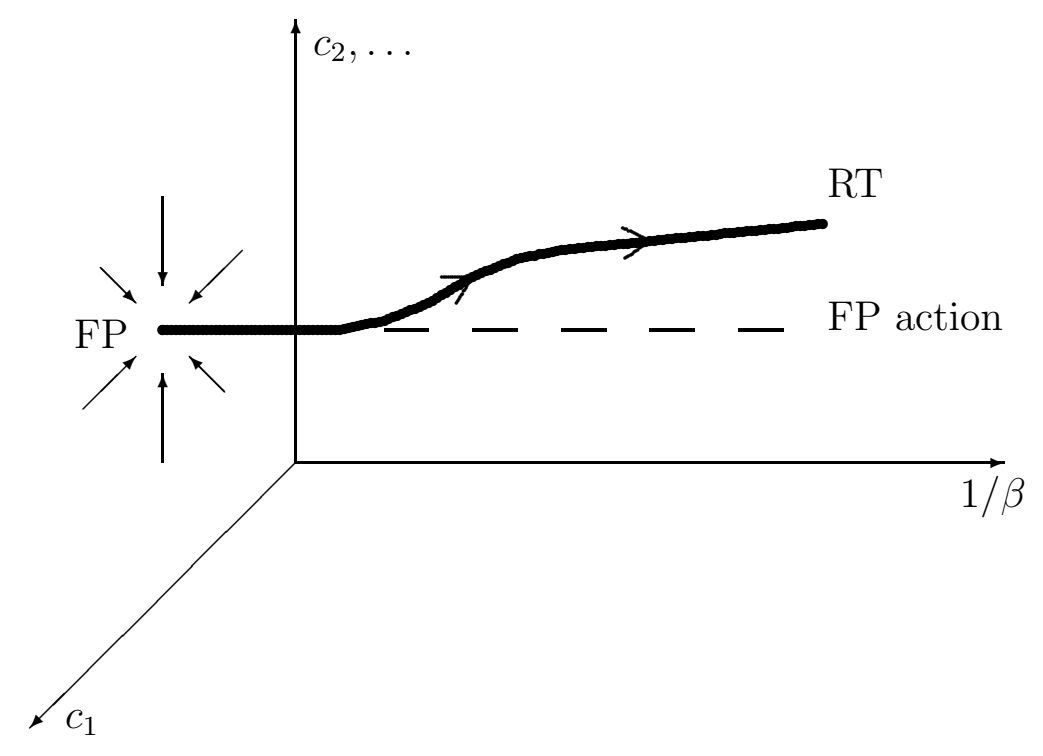

Figure 2: Flow of the couplings under RG transformation in the $\mathrm{O}(N)$ non-linear $\sigma-$ model

neighbourhood of the FP by (infinitely many steps of) exact RG transformations. Since each step increases the lattice unit by a factor of 2 , any distance at the given $\beta$ (even 1 lattice unit) corresponds to a long distance close to the FP. The infinitesimal neighbourhood of the FP is in the continuum limit, there are no cut-off effects there at long distances. On the other hand, for all the questions which can be formulated in terms of the degrees of freedom after the transformation we get the same answer as before it. So, there are no lattice artefacts at the given $\beta$ on the RT at any distances.

Since a RG step is a non-critical problem, the FP-action and the actions on the RT in general are expected to be local [5, 6]. Locality, however, allows nonnegligible interactions over several lattice units in the action. For practical reasons we neeed more than locality. For practical applicability we have to answer positively the following questions: i. Is it possible to determine $\mathcal{A}^{*}(\mathbf{S})$ to a good precision? ii. Is $\mathcal{A}^{*}(\mathbf{S})$ of sufficiently short ranged? Is the structure of $\mathcal{A}^{*}$ simple enough allowing a parametrization where the number of couplings remains relatively low, $\mathrm{O}(10-100)$ ? iii. Questions i. and ii. for the points of the RT.

Our pilot study shows that for the non-linear $\sigma$-model, using a properly chosen $\mathrm{RG}$ transformation, the answer to these questions is 'yes', even if we go down to small correlation lengths. The determination of $\mathcal{A}^{*}(\mathbf{S})$ is a saddle point problem which requires minimalization over classical fields. The $\mathrm{FP}, \mathcal{A}^{*}(\mathbf{S})$ is the perfect classical theory on the lattice: it has stable instanton solutions whose action is independent of the size $\rho$ of the instanton (scale invariance). This should be the 
same in $d=4$ gauge theories also. This fact might open new ways in the study of topological effects on the lattice. The range of interaction in $\mathcal{A}^{*}(\mathbf{S})$ depends on the RG transformation. By a proper choice of the block-transformation $\mathcal{A}^{*}(\mathbf{S})$ becomes surprisingly short ranged. $\mathcal{A}^{*}(\mathbf{S})$ contains multispin couplings also but its structure is relatively simple. With $\mathrm{O}(20)$ couplings an excellent parametrization can be obtained which works even on coarse configurations, i.e. at small correlation lengths. The problems related to $\mathcal{A}^{*}(\mathbf{S})$ can be solved partly analytically, which is a special bonus in asymptotically free theories.

If $\beta$ is not very large, the $\mathrm{FP}$-action $\mathcal{A}_{F P}=\beta_{F P} \mathcal{A}^{*}(\mathbf{S})$ is not a perfect action. It is, however, the perfect classical action and we expect that the lattice artefacts are significantly reduced. Actually, we were not able to identify any cut-off effects when simulating $\mathcal{A}_{F P}$ in our pilot study. The lattice artefacts in the problem of the running coupling in Fig. 1 can be used, for example, to illustrate how well the FP-action performs. As Fig. 3 shows, even the coarsest discretization $L / a=5$ gives a result which is consistent with the final extrapolated value. Let us emphasize at this point that $\mathcal{A}_{F P}$ has nothing to do with perturbation theory.

The FP-action is 'almost perfect', which suggests that the RT runs close to the $\mathrm{FP}$-action even at moderate $\beta$-values. In this case the blocked $\mathrm{FP}$-action will converge rapidly towards the RT. We illustrate on an explicit numerical experiment that the problem to find and parametrize the action after a block-transformation at finite $\beta$ is feasible. In traditional Monte Carlo simulations one goes deep in the continuum limit, simulates large lattices and fights with memory problems and critical slowing down. Our message is: do careful calculations on small lattices instead.

There remained many interesting problems even in this $d=2$ model which we did not touch: an explicit study of the classical solutions, the possibility of using strong coupling expansion rather than Monte Carlo simulations for the perfect action at small correlation lengths and, most notably, the problem of constructing currents corresponding to the perfect action.

The idea of constructing improved actions using RG ideas is old [4, [0]. But, perhaps because the problem was considered as not really feasible, already at the start crude approximations and assumptions were introduced. We would like to show here that - due to the specific properties of asymptotically free theories one can get close to the dream of a perfect regularization. First, finding the FP is a relatively easy classical problem. This is a significant advantage over other statistical models, e.g. the $d=2$ Ising model [24]. Second, even a simple optimalization makes the FP-action short ranged. Third, the FP-action, which is the perfect classical action, performs very well even at small correlation lengths. This feature makes it a good first approximation in finding the RT.

Earlier RG and Monte Carlo RG studies 8, 9] revealed many analogies between 
$d=4$ Yang-Mills theories and $d=2$ asymptotically free spin models. This gives a hope that the results of this paper can be generalized. Of course, the final goal is to treat full QCD where the cut-off effects are even more troublesome. The formalism can be extended to fermions. The free fermion problem has been discussed in [10], but the real hard work is to be done.

We should mention that the physical picture in the multigrid-RG program as formulated by Mack [23] has close analogies to that discussed here.

\section{The fixed point and the fixed point action}

\subsection{The equation for $\mathcal{A}^{*}$}

We shall define the RG transformation in the model in eq. (1) as follows. The square lattice is divided into $2 \times 2$ blocks and to every block we associate a block spin $\mathbf{R}_{n_{B}}$, which is a certain average of the four original spins in the block. We shall denote the points of the original and the block lattice by $n$ and $n_{B}$, respectively. The spins $\mathbf{S}_{n}$ and $\mathbf{R}_{n_{B}}$ are normalized $\mathrm{O}(N)$ vectors. The $\mathrm{RG}$ transformation is defined as

$$
\begin{aligned}
& \mathrm{e}^{-\beta^{\prime} \mathcal{A}^{\prime}(\mathbf{R})}=\int_{\mathbf{S}} \exp \left\{-\beta \mathcal{A}(\mathbf{S})+\sum_{n_{B}}\left[P \mathbf{R}_{n_{B}} \sum_{n \in n_{B}} \mathbf{S}_{n}-\ln Y_{N}\left(P\left|\sum_{n \in n_{B}} \mathbf{S}_{n}\right|\right)\right]\right\} \\
& \int_{\mathbf{S}}=\prod_{n} \int d \mathbf{S}_{n} \delta\left(\mathbf{S}_{n}^{2}-1\right)
\end{aligned}
$$

where $P$ is a parameter of the transformation, while the normalization factor $Y_{N}(z)$ assures that the partition function remains unchanged up to an irrelevant constant:

$$
\int d \mathbf{R} \delta\left(\mathbf{R}^{2}-1\right) \mathrm{e}^{\mathbf{R b}}=\text { const } \cdot Y_{N}(|\mathbf{b}|)
$$

The function $Y_{N}(z)$ is related to the modified Bessel function (some of its properties are summarized in Appendix $\mathrm{F}$ in [11], for example), specifically $Y_{3}(z) \sim$ $\sinh (\mathrm{z}) / \mathrm{z}$. For $P \rightarrow \infty$, the block-transformation goes over to a $\delta$-function giving $\mathbf{R}_{n_{B}}=\sum_{n \in n_{B}} \mathbf{S}_{n} /\left|\sum_{n \in n_{B}} \mathbf{S}_{n}\right|$. We keep, however, $P$ finite and write

$$
P=\beta \cdot[\kappa+\mathrm{O}(1 / \beta)],
$$

where $\kappa$ is a free parameter. Similar RG transformations were considered before in Monte Carlo RG studies [12]. The coupling $\beta$ is defined by the requirement that on very smooth configurations the action goes over to the form in eq. (2) (see also section 2.4). 
In the limit $\beta \rightarrow \infty$, eq. (3) can be written as

$$
\mathrm{e}^{-\beta^{\prime} \mathcal{A}^{\prime}(\mathbf{R})}=\int_{\mathbf{S}} \exp \left(-\beta\left\{\mathcal{A}(\mathbf{S})-\kappa \sum_{n_{B}}\left[\mathbf{R}_{n_{B}} \sum_{n \in n_{B}} \mathbf{S}_{n}-\left|\sum_{n \in n_{B}} \mathbf{S}_{n}\right|\right]\right\}\right),
$$

where $\beta^{\prime}=\beta-\mathrm{O}(1)$, due to asymptotic freedom. Eq. (6) is a saddle point problem in this limit, giving

$$
\mathcal{A}^{\prime}(\mathbf{R})=\min _{\{\mathbf{S}\}}\left\{\mathcal{A}(\mathbf{S})-\kappa \sum_{n_{B}}\left[\mathbf{R}_{n_{B}} \sum_{n \in n_{B}} \mathbf{S}_{n}-\left|\sum_{n \in n_{B}} \mathbf{S}_{n}\right|\right]\right\} .
$$

The FP of the transformation is determined by the equation

$$
\mathcal{A}^{*}(\mathbf{R})=\min _{\{\mathbf{S}\}}\left\{\mathcal{A}^{*}(\mathbf{S})-\kappa \sum_{n_{B}}\left[\mathbf{R}_{n_{B}} \sum_{n \in n_{B}} \mathbf{S}_{n}-\left|\sum_{n \in n_{B}} \mathbf{S}_{n}\right|\right]\right\} .
$$

As we discussed at length in the Introduction, the $\mathrm{FP}$-action $\beta \mathcal{A}^{*}(\mathbf{R})$ - although it is not 'perfect' at finite $\beta$ - is an excellent starting action for further studies even at moderate $\beta$-values (at moderate correlation lengths). So, we shall need $\mathcal{A}^{*}(\mathbf{R})$ for strongly fluctuating configurations also. Observe that eq. (6) reduces to the saddle point equation eq. (7) for any configuration $\mathbf{R}$. If the configuration $\mathbf{R}$ is strongly fluctuating, then the minimizing configuration $\mathbf{S}$ will not be smooth either. In general, eqs. (7),(8) and their solutions have nothing to do with perturbation theory.

Eq. (8) for $\mathcal{A}^{*}$ is a highly non-trivial equation. Some of the important properties of the solution can be obtained, however, without solving the equation explicitly.

\section{$2.2 \quad \mathcal{A}^{*}$ as a perfect classical theory on the lattice}

Using eq. (8) it is easy to show the following

\section{Statement}

If the configuration $\{\mathbf{R}\}$ satisfies the $\mathrm{FP}$ classical equations (i.e. the classical equations corresponding to $\mathcal{A}^{*}$ ) and it is a local minimum of $\mathcal{A}^{*}(\mathbf{R})$ (allowing also for zero modes) then the configuration $\{\mathbf{S}(\mathbf{R})\}$ on the finer lattice which minimizes the right hand side of eq. (8) satisfies the FP equations as well. In addition, the value of the action remains unchanged, $\mathcal{A}^{*}(\mathbf{S}(\mathbf{R}))=\mathcal{A}^{*}(\mathbf{R})$.

\section{Proof}

Since $\{\mathbf{R}\}$ is a solution of the classical equations of motion, $\delta \mathcal{A}^{*} / \delta \mathbf{R}=0$, the configuration $\{\mathbf{S}\}=\{\mathbf{S}(\mathbf{R})\}$ should satisfy the equation

$$
\sum_{n \in n_{B}} \mathbf{S}_{n}=\lambda_{n_{B}} \mathbf{R}_{n_{B}}
$$

\footnotetext{
${ }^{3}$ We are indebted to Uwe Wiese who raised our attention to the classical solutions in this context.
} 
for any $n_{B}$. Here $\lambda_{n_{B}} \geq 0$ since we excluded the case when $\mathcal{A}^{*}(\mathbf{R})$ is a local maximum in $\mathbf{R}_{n_{B}}$.

Since the expression

$$
\kappa \sum_{n_{B}}\left[\left|\sum_{n \in n_{B}} \mathbf{S}_{n}\right|-\mathbf{R}_{n_{B}} \sum_{n \in n_{B}} \mathbf{S}_{n}\right]
$$

in eq. (8) takes its absolute minimum (zero) on the configuration $\{\mathbf{S}(\mathbf{R})\}$ satisfying eq. (9), $\{\mathbf{S}(\mathbf{R})\}$ is also a stationary point of $\mathcal{A}^{*}$,

$$
\left.\frac{\delta \mathcal{A}^{*}(\mathbf{S})}{\delta \mathbf{S}}\right|_{\mathbf{S}=\mathbf{S}(\mathbf{R})}=0,
$$

with the same value of the action, $\mathcal{A}^{*}(\mathbf{S}(\mathbf{R}))=\mathcal{A}^{*}(\mathbf{R})$, what we wanted to show.

According to this Statement, to any solution $\{\mathbf{R}\}$ (which is a local minimum) with a characteristic size $\rho$, there exists another solution $\{\mathbf{S}(\mathbf{R})\}$ of size $2 \rho$ with exactly the same value of the action $\mathcal{A}^{*}$. It is natural to assume that this solution on the finer lattice is also a local minimum. Specifically, for $N=3$ this observation implies that if $\mathcal{A}^{*}$ has an instanton solution of size $\rho$ then there exist instanton solutions of size $2 \rho, 2^{2} \rho, \ldots, 2^{k} \rho, \ldots$ with the value of the action being exactly $4 \pi$ for all these instantons. The value $4 \pi$ follows from the fact that very large instantons are smooth on the lattice and then any valid lattice representation of eq. (2) gives the continuum value.

The standard action has classical solutions of antiferromagnetic character - e.g. when one of the spins points downwards in a background of upward pointing spins - which are smooth local maxima in some of the spins. We expect, although we are not able to prove, that $\mathcal{A}^{*}$ does not have such solutions. For example, the configuration mentioned above is not a solution since $\delta \mathcal{A}^{*}(\mathbf{R}) / \delta \mathbf{R}$ does not vanish at $\vartheta=\pi$, as shown in Fig. 6.

It was observed earlier in [13] that using an approximate RG improved action the stability of instantons can be increased significantly. This result is in accordance with the Statement above.

It is important to observe that the reverse of the Statement is not true: if the configuration $\left\{\mathbf{S}_{n}\right\}$ is a solution, then the configuration $\left\{\mathbf{R}_{n_{B}}\right\}$, where $\mathbf{R}_{n_{B}}=$ $\sum_{n \in n_{B}} \mathbf{S}_{n} /\left|\sum_{n \in n_{B}} \mathbf{S}_{n}\right|$, is not necessarily a solution. The proof fails because for this configuration $\left\{\mathbf{R}_{n_{B}}\right\}$ the minimizing configuration $\{\mathbf{S}(\mathbf{R})\}$ in eq. (8) is not necessarily equal to $\left\{\mathbf{S}_{n}\right\}$ itself. One can show that $\left\{\mathbf{S}_{n}\right\}$ is a minimum but not always the absolute minimum. Actually, this is the mechanism which prevents the existence of arbitrarily small instanton solutions on the lattice [14].

With respect to the classical solutions, $\mathcal{A}^{*}$ has the same scale symmetry as the continuum action. We shall give later further arguments which show that it is a perfect classical lattice representation of the continuum action. 


\subsection{Parametrization}

Eq. (8) defines the value of the $\mathrm{FP}, \mathcal{A}^{*}$ for any configuration $\mathbf{R}$ and, as we shall discuss later, one can advise numerical procedures which give this value to high precision. However, if we want to use the FP action $\beta_{F P} \mathcal{A}^{*}$ in numerical simulations we can not avoid to parametrize $\mathcal{A}^{*}$ by a (limited) number of coupling constants. We can write in general

$$
\begin{aligned}
\mathcal{A}^{*}(\mathbf{S})= & -\frac{1}{2} \sum_{n, r} \rho(r)\left(1-\mathbf{S}_{n} \mathbf{S}_{n+r}\right) \\
& +\sum_{n_{1}, n_{2}, n_{3}, n_{4}} c\left(n_{1}, n_{2}, n_{3}, n_{4}\right)\left(1-\mathbf{S}_{n_{1}} \mathbf{S}_{n_{2}}\right)\left(1-\mathbf{S}_{n_{3}} \mathbf{S}_{n_{4}}\right)+\ldots
\end{aligned}
$$

where the summations go over all the lattice points. It is a significant help in the parametrization and optimalization problem that the first two functions $\rho$ and $c$ in eq. (12) can be calculated directly. We shall discuss this problem first.

\subsection{The determination of $\rho(r)$}

Consider a configuration $\mathbf{R}$ where the spins fluctuate around the first axes:

$$
\mathbf{R}_{n_{B}}=\left(\begin{array}{c}
\sqrt{1-\vec{\chi}_{n_{B}}^{2}} \\
\vec{\chi}_{n_{B}}
\end{array}\right)
$$

where $\vec{\chi}_{n_{B}}$ has $(N-1)$ components, and $\left|\vec{\chi}_{n_{B}}\right| \ll 1$. In this case, the saddle-point configuration in eq. (8) will also fluctuate around the first axes, so we can write

$$
\mathbf{S}_{n}=\left(\begin{array}{c}
\sqrt{1-\vec{\pi}_{n}^{2}} \\
\vec{\pi}_{n}
\end{array}\right)
$$

where $\left|\vec{\pi}_{n}\right| \ll 1$. The terms quadratic in $\vec{\pi}$ and $\vec{\chi}$ give a closed equation for $\rho$ when eqs. (8) and (12) are used:

$$
\frac{1}{2} \sum_{n_{B}, r_{B}} \rho\left(r_{B}\right) \vec{\chi}_{n_{B}} \vec{\chi}_{n_{B}+r_{B}}=\min _{\{\vec{\pi}\}}\left\{\frac{1}{2} \sum_{n, r} \rho(r) \vec{\pi}_{n} \vec{\pi}_{n+r}+2 \kappa \sum_{n_{B}}\left(\vec{\chi}_{n_{B}}-\frac{1}{4} \sum_{n \in n_{B}} \vec{\pi}_{n}\right)^{2}\right\} .
$$

The equation for $\rho$ is independent of $N$ as far as $N>1$. Eq. (15) is just the FP equation of a free scalar theory with a Gaussian block-transformation:

$$
\begin{aligned}
& c \cdot \exp \left\{-\frac{1}{2} \sum_{n_{B}, r_{B}} \rho\left(r_{B}\right) \vec{\chi}_{n_{B}} \vec{\chi}_{n_{B}+r_{B}}\right\}= \\
& \prod_{n} \int d \vec{\pi}_{n} \exp \left\{-2 \kappa \sum_{n_{B}}\left(\vec{\chi}_{n_{B}}-\frac{1}{4} \sum_{n \in n_{B}} \vec{\pi}_{n}\right)^{2}-\frac{1}{2} \sum_{n, r} \rho(r) \vec{\pi}_{n} \vec{\pi}_{n+r}\right\} .
\end{aligned}
$$


Eqs. (15) and (16) are equivalent: Gaussian integrals can be replaced by minimalizations. The free field problem has been studied and solved in [15] a long time ago. In Fourier space the solution has the form

$$
\frac{1}{\tilde{\rho}(q)}=\sum_{l=-\infty}^{+\infty} \frac{1}{(q+2 \pi l)^{2}} \prod_{i=0}^{1} \frac{\sin ^{2}\left(q_{i} / 2\right)}{\left(q_{i} / 2+\pi l_{i}\right)^{2}}+\frac{1}{3 \kappa}
$$

where the summation is over the integer vector $l=\left(l_{0}, l_{1}\right),(q+2 \pi l)^{2}=\left(q_{0}+2 \pi l_{0}\right)^{2}+$ $\left(q_{1}+2 \pi l_{1}\right)^{2}$ and

$$
\rho(n)=\int_{-\pi}^{\pi} \frac{d^{2} q}{(2 \pi)^{2}} \mathrm{e}^{i q n} \tilde{\rho}(q)
$$

The normalization of $\rho$ is fixed by demanding $\tilde{\rho}(q) \rightarrow q^{2}$ for small $q$. In configuration space this corresponds to $\sum_{r} \rho(r) r^{2}=-4$. We fix the value of $\rho(0)$ by the convention $\sum_{r} \rho(r)=0$.

There is an elegant, simple way to derive eq. (17) which we discuss below, since it might simplify the analogous problem for gauge and fermion fields also. A similar trick was used in [16] in an approximate $\mathrm{RG}$ calculation in the large $-N$ limit with $\delta$-function blocking.

The observation is that in a free field theory the two-point function is directly related to the action. On the other hand, the two-point function at the FP can be calculated easily. From eq. (16) it follows

$$
\left\langle\chi_{n_{B}} \chi_{n_{B}^{\prime}}\right\rangle=\left(\frac{1}{4}\right)^{2} \sum_{\substack{n \in n_{B} \\ n^{\prime} \in n_{B}^{\prime}}}\left\langle\pi_{n} \pi_{n^{\prime}}\right\rangle+\frac{1}{4 \kappa} \delta_{n_{B} n_{B}^{\prime}} .
$$

Using this relation recursively, after $j \mathrm{RG}$ steps we get

$$
\left\langle\chi_{n_{B}} \chi_{n_{B}^{\prime}}\right\rangle=\left(\frac{1}{4}\right)^{2 j} \sum_{\substack{n \in n_{B} \\ n^{\prime} \in n_{B}^{\prime}}}\left\langle\pi_{n} \pi_{n^{\prime}}\right\rangle+\frac{1}{4 \kappa}\left(1+\frac{1}{4}+\frac{1}{4^{2}}+\ldots+\frac{1}{4^{j}}\right) \delta_{n_{B} n_{B}^{\prime}} .
$$

where $\chi$ is the block-field after $j$ steps, and the block $n_{B}$ contains $4^{j}$ original lattice points. Taking the $j \rightarrow \infty$ limit, and considering the original lattice infinitely fine, the summations in the first term on the right-hand side of eq. (20) go over to integrals. The integrals are over a block $(a \times a)$ centered around the points $n_{B}$ and $n_{B}^{\prime}$, respectively. For the two-point function on the infinitely fine lattice we can use the standard $1 / k^{2}$ propagator. Taking $a=1$, and simplifying the notations we get

$$
\left\langle\chi_{n} \chi_{n^{\prime}}\right\rangle=\int_{-1 / 2}^{1 / 2} d^{2} x \int_{-1 / 2}^{1 / 2} d^{2} x^{\prime} \int_{-\infty}^{\infty} \frac{d^{2} k}{(2 \pi)^{2}} \frac{\mathrm{e}^{i k\left(n+x-n^{\prime}-x^{\prime}\right)}}{k^{2}}+\frac{1}{3 \kappa} \delta_{n n^{\prime}} .
$$

After many RG steps the system runs to the FP (taking the mass to be zero at the beginning, only irrelevant directions remain), therefore

$$
\left\langle\chi_{n} \chi_{n^{\prime}}\right\rangle=\int_{-\pi}^{\pi} \frac{d^{2} q}{(2 \pi)^{2}} \frac{\mathrm{e}^{q\left(n-n^{\prime}\right)}}{\tilde{\rho}(q)} .
$$


Dividing the $k$-integration into an integration and summation by introducing the integer vector $l=\left(l_{0}, l_{1}\right)$

$$
k_{i}=q_{i}+2 \pi l_{i}, \quad q_{i} \in(-\pi, \pi), \quad i=0,1,
$$

and performing the integrals over $x$ and $x^{\prime}$, the result in eq. (17) follows. Using similar steps, the two-point function in configuration space has been obtained for $\delta$-function blocking by Iwasaki [四] and by Gawedzki and Kupiainen [17] also.

Let us remark that the $1 / 4$ factor in the block transformation of a free theory in eq. (16) is replaced by $b=2^{-(d+2) / 2}$ in $d$ dimensions. Only this choice leads to a FP [5, 15]. In the non-linear $\sigma$-model, the factor $1 / 4$ in the last term in eq. (15) is fixed. That these two factors coincide for $d=2$ is related to the fact that in two dimensions (and only in this case) the non-linear $\sigma$-model is asymptotically free.

\subsection{The properties of $\rho(r)$; the perfect Laplace operator on the lattice}

The properties (most notably the range of interaction) of $\rho(r)$ depend on the free parameter $\kappa$. The choice $\kappa \approx 2$. is optimal. For $\kappa=2, \rho(r)$ decays very rapidly with growing $|r|$, like $\sim \exp (-3.44|r|)$, Fig. 4. For this choice $\rho(r)$ is strongly dominated by the nearest neighbour and diagonal couplings while the couplings at distance $>1$ are already small. As Table 1 shows, $\rho(3,3)$ is, for example, 5 orders of magnitude smaller than the nearest neighbour coupling. The $\kappa=2$ value is distinguished also by the fact that in $d=1$ (or, equivalently, in any $d$ on configurations which depend only on one coordinate) this choice leads to a simple nearest neighbour action. Fig. 4 also shows that the choice $\kappa=\infty$ (corresponding to a block transformation with $\delta$-function) gives a considerably larger interaction range, $\rho(r) \sim \exp (-1.45|r|)$.

A free field theory defined in terms of $\rho$ on the lattice gives a cut-off independent account of the physical properties of the theory. The spectrum is exact. In the continuum the energy of an excitation with momentum $k_{1}$ is $E\left(k_{1}\right)=\left|k_{1}\right|$, where $k_{1} \in(-\infty, \infty)$ (massless relativistic free particle). Using eq. (17) one can check easily that the lattice field theory with $\rho$ has exactly this spectrum. (A possible method is to calculate the two-point function at large time-separation.) Although $q_{1}$ in eq. (17) lies in $(-\pi, \pi)$ only, the full spectrum is reproduced because for every given $q_{1}$ there are infinitely many energy eigenstates. This is related to the summation over $l$ in eq. (17).

Fig. 5 compares the predictions for the energy spectrum of $\rho$ (exact) to those of the standard lattice regularization, Symanzik tree level improved action and the approximation where the $\rho(1,0)$ and $\rho(1,1)$ couplings are kept only. f Already this crudest approximation performs very well.

\footnotetext{
${ }^{4}$ We are indebted to M. Blatter for his help on Fig. 5.
} 


\begin{tabular}{|c|c||c|c|}
\hline$r$ & $\rho(r)$ & $r$ & $\rho(r)$ \\
\hline$(1,0)$ & -0.61802 & $(4,0)$ & $-2.632 \cdot 10^{-6}$ \\
$(1,1)$ & -0.19033 & $(4,1)$ & $7.064 \cdot 10^{-7}$ \\
$(2,0)$ & $-1.998 \cdot 10^{-3}$ & $(4,2)$ & $1.327 \cdot 10^{-6}$ \\
$(2,1)$ & $-6.793 \cdot 10^{-4}$ & $(4,3)$ & $-7.953 \cdot 10^{-7}$ \\
$(2,2)$ & $1.625 \cdot 10^{-3}$ & $(4,4)$ & $6.895 \cdot 10^{-8}$ \\
$(3,0)$ & $-1.173 \cdot 10^{-4}$ & $(5,0)$ & $-8.831 \cdot 10^{-8}$ \\
$(3,1)$ & $1.942 \cdot 10^{-5}$ & $(5,1)$ & $3.457 \cdot 10^{-8}$ \\
$(3,2)$ & $5.232 \cdot 10^{-5}$ & $(5,2)$ & $3.491 \cdot 10^{-8}$ \\
$(3,3)$ & $-1.226 \cdot 10^{-5}$ & $(5,3)$ & $-3.349 \cdot 10^{-8}$ \\
& & $(5,4)$ & $8.408 \cdot 10^{-9}$ \\
& & $(5,5)$ & $-1.657 \cdot 10^{-10}$ \\
\hline
\end{tabular}

Table 1: The couplings of the quadratic two-spin interaction terms at a distance $r=\left(r_{0}, r_{1}\right)$ for the optimal choice of the block transformation with $\kappa=2$. Note that in our convention, for the standard action the only non-vanishing entry in this list would be $\rho_{S T}(1,0)=-1$.

The finite difference operator $\left.\Delta_{L} \Phi\right)_{n}=-\sum_{r} \rho(r) \Phi_{n+r}$ is a perfect discretization of the continuum Laplacian. Let $\phi(x)$ be a field in the continuum satisfying the Laplace equation

$$
\Delta \phi(x)=0 .
$$

Lay a lattice on the $d=2$ continuum plane with lattice unit 1 and define on the lattice point $n$ a lattice field

$$
\Phi_{n}=\int_{-\frac{1}{2}}^{\frac{1}{2}} d^{2} x \phi(n+x)
$$

The field $\Phi_{n}$ satisfies

$$
\Delta_{L} \Phi=0,
$$

and gives the same action as $\phi(x)$ :

$$
-\int d^{2} x \phi \Delta \phi=-\sum_{n} \Phi_{n}\left(\Delta_{L} \Phi\right)_{n}
$$

\subsection{The determination of $c\left(n_{1}, n_{2}, n_{3}, n_{4}\right)$}

In order to obtain the coefficients $c(.$.$) we have to expand eqs. (8.,12) up to quartic$ order in $\vec{\pi}$ and $\vec{\chi}$. In quadratic order, discussed before, the minimizing $\pi$ configura- 
tion in eq. (8) is linear in $\chi$ :

$$
\vec{\pi}_{n}=\sum_{n_{B}^{\prime}} M\left(n, n_{B}^{\prime}\right) \vec{\chi}_{n_{B}^{\prime}}
$$

where the matrix $M$ is short ranged (if $n$ is in the block $n_{B}$ then only small $\left|n_{B}-n_{B}^{\prime}\right|$ gives significant contribution in the sum in eq. (28)) and can be obtained explicitly when solving the free field problem [15]. In order to obtain the $\chi$ dependence of the right-hand side of eq. (8) up to quartic order it is sufficient to substitute the minimizing value of $\pi$ up to linear order, eq. (28). The cubic corrections to eq. (28) give $\mathrm{O}\left(\chi^{6}\right)$ terms only in eq. (8).

In quartic order eq. (8) can be written in the form

$$
\sum_{n_{B}, n_{B}^{\prime}, n_{B}^{\prime \prime}, n_{B}^{\prime \prime \prime}} A\left(n_{B}, n_{B}^{\prime} ; n_{B}^{\prime \prime}, n_{B}^{\prime \prime \prime}\right)\left(\vec{\chi}_{n_{B}} \vec{\chi}_{n_{B}^{\prime}}\right)\left(\vec{\chi}_{n_{B}^{\prime \prime}} \vec{\chi}_{n_{B}^{\prime \prime \prime}}\right)=0,
$$

where the matrix $A$ depends linearly on $c(\ldots)$ and is symmetric under $n_{B} \leftrightarrow n_{B}^{\prime}$, $n_{B}^{\prime \prime} \leftrightarrow n_{B}^{\prime \prime \prime}$, and $\left(n_{B}, n_{B}^{\prime}\right) \leftrightarrow\left(n_{B}^{\prime \prime}, n_{B}^{\prime \prime \prime}\right)$. Its form is not very illuminating and can be easily derived, so we do not quote it here explicitly. Although the matrix $A$ has no explicit $N$-dependence, the solution $c(\ldots)$ distinguishes between $N \geq 3$ and $N=2$. For $N \geq 3$ eq. (29) implies $A=0$, while for $N=2 \chi$ is a 1 -component vector and eq. (29) gives

$$
A\left(n_{B}, n_{B}^{\prime} ; n_{B}^{\prime \prime}, n_{B}^{\prime \prime \prime}\right)+A\left(n_{B}^{\prime \prime}, n_{B}^{\prime} ; n_{B}, n_{B}^{\prime \prime \prime}\right)=0, \quad(N=2) .
$$

These equations can be solved for $c(.$.$) by a rapidly converging iterative proce-$ dure. The short range nature of $\rho(r)$ for $\kappa=2$ is inherited by $c(\ldots)$, as expected. Table 2 enumerates those couplings with $|c(\ldots)|>5 \cdot 10^{-5}$.

\subsection{The numerical procedure to solve the FP equation}

For a given configuration $\{\mathbf{R}\}$, eqs. $(17, \mathbb{8})$ can be solved numerically for the FP value $\mathcal{A}^{*}(\mathbf{R})$. Calculating $\mathcal{A}^{*}(\mathbf{R})$ for a large number of different $\{\mathbf{R}\}$ configurations, the important couplings (beyond those in $\rho(r)$ and $c(.$.$) ) can be found by a fitting$ procedure. The finite size distortion on the couplings will be small if the lattice on which $\{\mathbf{R}\}$ is defined is larger than the range of interaction in $\mathcal{A}^{*}$. Since the later is small for $\kappa=2$ (see $\rho(r)$ and $c(\ldots)$, and further discussion in 2.8) this requirement is easy to satisfy. In this pilot study we worked on $5 \times 5$ periodic lattices when looking for the couplings of the $\mathrm{FP}, \mathcal{A}^{*}$ numerically.

We worked out two different numerical procedures to calculate $\mathcal{A}^{*}(\mathbf{R})$ for a given configuration $\{\mathbf{R}\}$. Both were based on iterating eq. (7). For a process with $k$ iterations we define the lattice sizes $L^{(0)}, L^{(1)}=L^{(0)} / 2, \ldots, L^{(k)}=L^{(0)} / 2^{k}$. The 


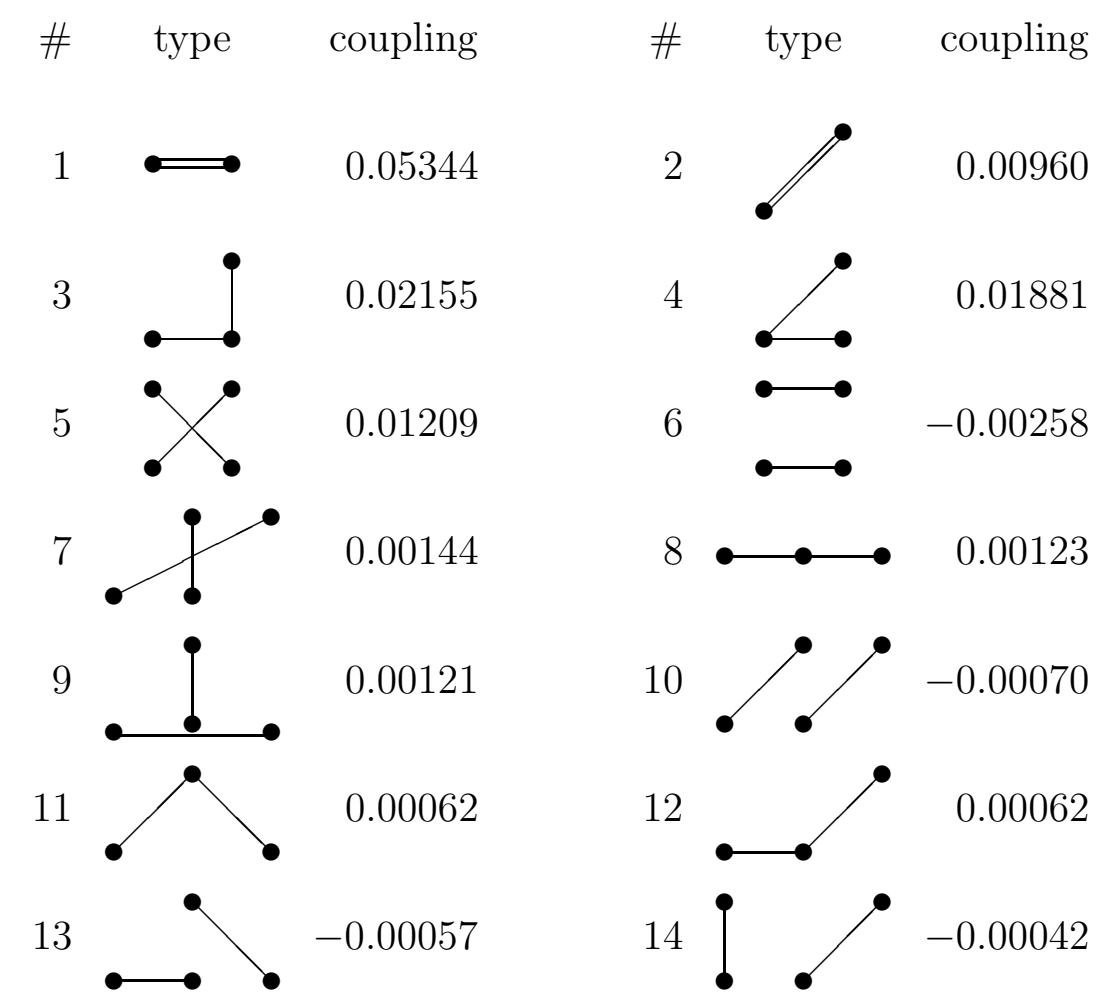

Table 2: The coefficients of the leading quartic terms $\left(1-\mathbf{S}_{n_{1}} \mathbf{S}_{n_{2}}\right)\left(1-\mathbf{S}_{n_{3}} \mathbf{S}_{n_{4}}\right)$ of $\mathcal{A}^{*}$. A term which is defined by the sites $n_{1}, n_{2}, n_{3}, n_{4}$ and the topology of the four-spin coupling occurs only once with these coefficients in the action. The numbers in this Table are multiples of $c\left(n_{1}, \ldots, n_{4}\right)$ in eq. (12). This symmetry factor is 4 for the couplings \#1,2 and 8 for the others. 
configuration $\{\mathbf{R}\}$ lives on the lattice of size $L^{(k)}(=5$, in our case), while we denote the spins on the $0^{t h}, 1^{s t}, \ldots,(k-1)^{t h}$ level by $\mathbf{S}_{n_{0}}^{(0)}, \mathbf{S}_{n_{1}}^{(1)}, \ldots, \mathbf{S}_{n_{k-1}}^{(k-1)}$. We get

$$
\begin{aligned}
& \mathcal{A}^{(k)}(\mathbf{R})=\min _{\left\{\mathbf{S}^{(0)}, \mathbf{S}^{(1)}, \ldots, \mathbf{S}^{(k-1)}\right\}}\left\{\mathcal{A}^{(0)}\left(\mathbf{S}^{(0)}\right)-\kappa \sum_{n_{1}}\left[\mathbf{S}_{n_{1}}^{(1)} \sum_{n_{0} \in n_{1}} \mathbf{S}_{n_{0}}^{(0)}-\left|\sum_{n_{0} \in n_{1}} \mathbf{S}_{n_{0}}^{(0)}\right|\right]\right. \\
& -\kappa \sum_{n_{2}}\left[\mathbf{S}_{n_{2}}^{(2)} \sum_{n_{1} \in n_{2}} \mathbf{S}_{n_{1}}^{(1)}-\left|\sum_{n_{1} \in n_{2}} \mathbf{S}_{n_{1}}^{(1)}\right|\right](31) \\
& \left.-\kappa \sum_{n_{k}}\left[\mathbf{R}_{n_{k}} \sum_{n_{k-1} \in n_{k}} \mathbf{S}_{n_{k-1}}^{(k-1)}-\left|\sum_{n_{k-1} \in n_{k}} \mathbf{S}_{n_{k-1}}^{(k-1)}\right|\right]\right\} .
\end{aligned}
$$

Here $\mathcal{A}^{(0)}$ is the zeroth order approximation for $\mathcal{A}^{*}$, while $\mathcal{A}^{(k)}(\mathbf{R})$ is the $k^{\text {th }}$ approximation for the number $\mathcal{A}^{*}(\mathbf{R})$. For $\mathcal{A}^{(0)}$ one might take even the standard action, but the convergence in $k$ will improve if a better approximation is used. (For example, one can use the results on $\rho(r)$ and $c(\ldots)$, or those of earlier numerical runs.) In eq. (31) $\sum_{n_{0} \in n_{1}} \mathbf{S}_{n_{0}}^{(0)}$ denotes a sum over the 4 spins in the block $n_{1}$, etc.

Note that eq. (31) and the way we solved it are strongly reminiscent to a multigrid procedure [23, 18].

In the first method the minimalization in $\mathbf{S}$ was performed locally and the lattices were swept through until $\mathcal{A}^{(k)}(\mathbf{R})$ became stable to a given precision. In the second method we used annealed cooling. The largest irrelevant eigenvalue of the FP is $1 / 4$ in agreement with the observed convergence rate in the number of blocking steps $k$. Even taking the crude approximation for $\mathcal{A}^{(0)}$ where only the nearest neighbour and diagonal couplings from $\rho(r)$ are kept, $\left|\mathcal{A}^{(4)}(\mathbf{R})-\mathcal{A}^{*}(\mathbf{R})\right| /\left|\mathcal{A}^{*}(\mathbf{R})\right|$ was estimated less than $\mathrm{O}\left(10^{-4}\right)$. The two different methods gave consistent results.

\subsection{The properties of $\mathcal{A}^{*}$ on coarse configurations}

On smooth configurations the first terms in eq. (12) dominate. These terms are short ranged and explicitly known. It is important to investigate, however, whether $\mathcal{A}^{*}$ remains short ranged even on coarse configurations. We need further hints on the general parametrization problem also.

In order to see how $\mathcal{A}^{*}(\mathbf{S})$ behaves as the relative angle between the spins is increased, we rotated a single spin $\mathbf{S}$ away from the $1^{\text {st }}$ axis by an angle $\vartheta$, while all the other spins pointed in the $1^{\text {st }}$ direction. The corresponding value of $\mathcal{A}^{*}$, which we denote by $\mathcal{A}_{1}(\vartheta)$, is shown in Fig. 6 . The curve is very simple and can be fitted easily if we parametrize it in powers of $\vartheta^{2} / 2$ rather than in powers of $(1-\cos \vartheta)$. This empirical observation is also supported by considering a quasi one-dimensional solution to the equations of motion, with $\mathbf{S}(n)=\left(\cos \vartheta n_{0}, \sin \vartheta n_{0}, 0\right)$, where the $\vartheta$ 


\begin{tabular}{|c|c|c|c|c|}
\hline$\vartheta$ & $\mathcal{A}_{1}(\vartheta)$ & $\mathcal{A}_{2}(\vartheta)$ & $\mathcal{A}_{1}(\vartheta)-\frac{1}{2} \mathcal{A}_{2}(\vartheta)$ & $-\rho(2,0) \vartheta^{2} / 2$ \\
\hline 0.09967 & 0.016094 & 0.032166 & 0.000011 & 0.000010 \\
$\pi / 4$ & 0.99558 & 1.98986 & 0.00065 & 0.00062 \\
$\pi / 2$ & 3.9339 & 7.8627 & 0.0025 & 0.0025 \\
2.678 & 10.9999 & 21.9863 & 0.0067 & 0.0072 \\
2.85 & 12.3352 & 24.6553 & 0.0075 & 0.0081 \\
\hline
\end{tabular}

Table 3: One and two spins (at a separation $(2,0)$ ) are rotated in a trivial background. The values of $\mathcal{A}^{*}$ for the corresponding configurations are given together with $\mathcal{A}_{1}(\vartheta)-\frac{1}{2} \mathcal{A}_{2}(\vartheta)$ which measures the direct $(2,0)$ interaction. The last column shows the contribution from the first term in eq. (12').

dependence of $A^{*}$ is exactly given by $\vartheta^{2} / 2$. So, we rewrite eq. (12) as

$$
\mathcal{A}(\mathbf{S})=-\frac{1}{2} \sum_{n, r} \rho(r) \frac{1}{2} \vartheta_{n, n+r}^{2}+\sum_{n_{1}, n_{2}, n_{3}, n_{4}} \bar{c}\left(n_{1}, n_{2}, n_{3}, n_{4}\right) \frac{1}{2} \vartheta_{n_{1}, n_{2}}^{2} \cdot \frac{1}{2} \vartheta_{n_{3}, n_{4}}^{2}+\ldots
$$

where $\vartheta_{n_{1}, n_{2}}$ is the angle between the spins $\mathbf{S}_{n_{1}}$ and $\mathbf{S}_{n_{2}}$. The function $\bar{c}$ is trivially related to $\rho$ and $c$ of eq. (12). Using this parametrization, the leading couplings of $\rho$ and $\bar{c}$ will dominate $\mathcal{A}^{*}$ even on coarse configurations. This is illustrated in Fig. 6, where a $d_{1} \vartheta^{2} / 2+d_{2}\left(\vartheta^{2} / 2\right)^{2}$ form, with $d_{1}$ and $d_{2}$ obtained from $\rho$ and $c$ in Tables 1,2 , agree very well with the data.

Next we wanted to check whether the distant couplings remain small even at large relative angles. In a trivial background (spins in the $1^{\text {st }}$ direction) we rotated 2 spins, which were at a distance $\Delta$ from each other, by an angle $\vartheta$ relative to the $1^{\text {st }}$ axes (keeping them parallel to each other). Denoting the corresponding value of $A^{*}$ by $A_{2}(\vartheta)$ it is easy to see that $A_{1}(\vartheta)-\frac{1}{2} A_{2}(\vartheta)$ is a measure of the direct coupling between the two spins. Table 3 gives the measured values at different $\vartheta$ values for distance $\Delta=(2,0)$ compared with $-\rho(2,0) \vartheta^{2} / 2$, the first, exactly known term in eq. (12'). The direct coupling remains small and very well given by this single term even at large angles.

\subsection{Fixing the couplings in this pilot study in $\mathrm{O}(3)$}

In the following we consider the $\mathrm{O}(3)$ model, $N=3$. We generated $\sim 500$ different $\mathbf{R}$ configurations and calculated the corresponding values of the $\mathrm{FP}, \mathcal{A}^{*}(\mathbf{R})$ using the minimalization procedure discussed in 2.7. Among these configurations there were smooth, but also quite coarse ones, typical for a correlation length of a few lattice units only. We made fits with up to 69 different couplings, taking them all short ranged, with the largest distance being $(2,2)$. 


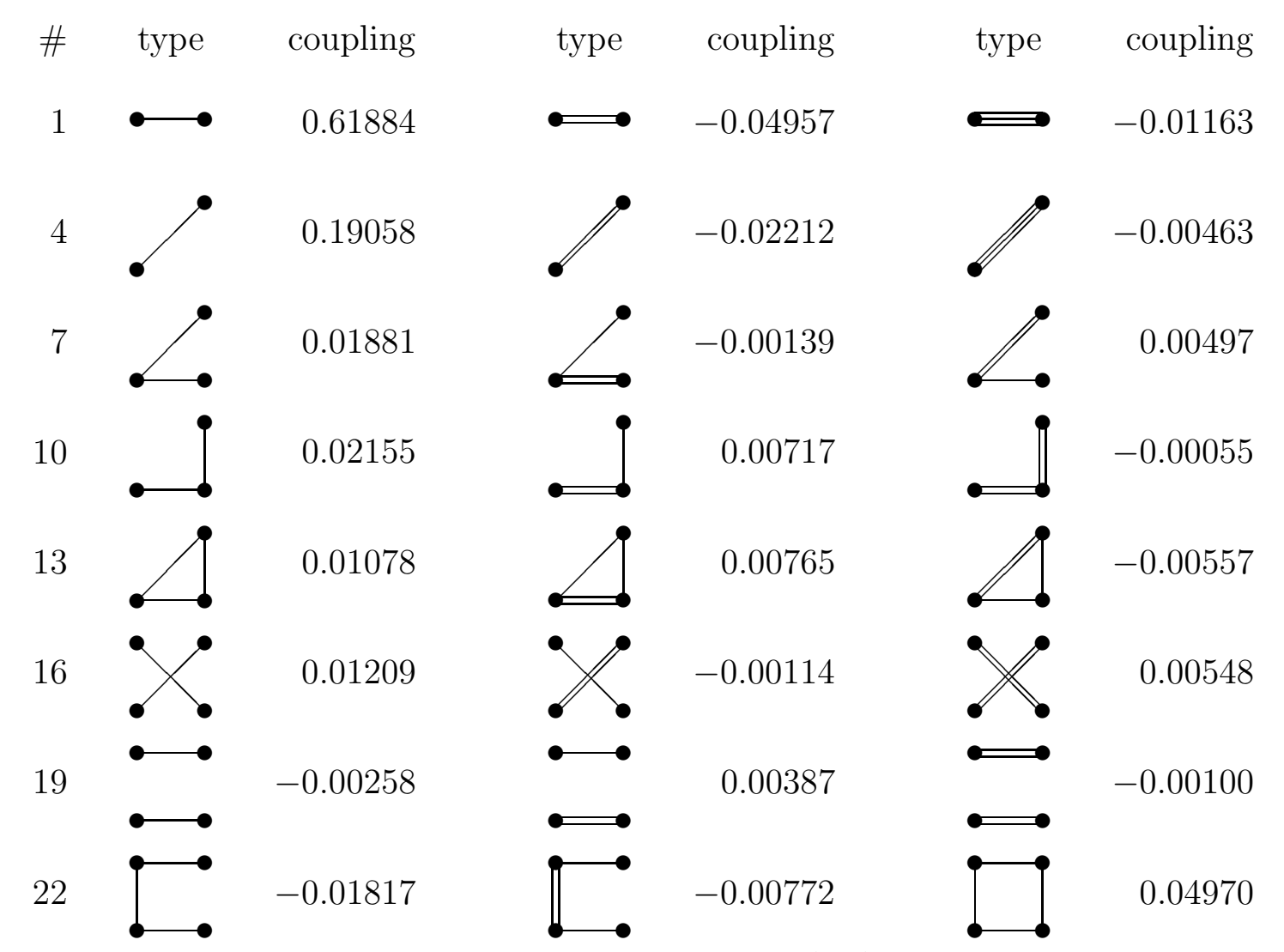

Table 4: The couplings used for the FP-action in eq. (12'). The notation is explained in the text. The coefficients of the quadratic and quartic interactions are calculated analytically, the higher order interactions are fitted.

Fig. 7 shows a fit with 24 couplings, 8 fixed analytically by $\rho$ and $c, 16$ fitted. The couplings and their fitted values are given in Table 4. The couplings \#1 and 4 are slightly renormalized to satisfy the condition $\sum_{r} \rho(r) r^{2}=-4$ exactly. In this fit all the couplings can be put on a $1 \times 1$ square. In Table 4 the couplings are given in a graphical notation. The coupling \#15, for example, multiplies the expression $\frac{1}{2} \vartheta_{A}^{2} \cdot \frac{1}{2} \vartheta_{B}^{2} \cdot\left(\frac{1}{2} \vartheta_{C}^{2}\right)^{2}$, where $\vartheta_{A}=\operatorname{acos}\left(\mathbf{S}_{1} \mathbf{S}_{2}\right), \vartheta_{B}=\operatorname{acos}\left(\mathbf{S}_{1} \mathbf{S}_{3}\right)$ and $\vartheta_{C}=\operatorname{acos}\left(\mathbf{S}_{2} \mathbf{S}_{3}\right)$. Here $\mathbf{S}_{1}, \mathbf{S}_{2}, \mathbf{S}_{3}$ are the 3 spins forming the triangle, $\mathbf{S}_{1}$ sitting at the $90^{\circ}$ corner.

As Fig. 7 shows the fit becomes poorer on coarse configurations (those have larger action values), in a few cases the error reaches $\sim 1 \%$, but for most of the configurations the error is much smaller. Including the $(2,0),(2,1)$ and $(2,2)$ couplings the fit becomes somewhat better, but for this pilot study we decided to use the 24-parameter fit in Table 4.

We want to emphasize two points here. Using 24 , or even twice as many couplings in a Monte Carlo simulation is not really a problem - at least in the spin model considered here. Although this action includes 3- and 4-spin interactions also, one can generalize the cluster Monte Carlo technique [19] for this case. Our cluster 
program runs $\sim 3$ times slower with 24 parameters than with the standard action. Relative to the gain we are looking for, this is irrelevant. The second remark concerns the numbers in Table 4. The fitted parameters are effective values, one must not associate significance to a single entry in this Table. Modifying somewhat the set of operators used in the fit, these entries would change, but the global quality of the fit would hardly be different.

\subsection{Results obtained by simulating the FP action}

The FP-action $\mathcal{A}_{F P}^{*}$ is defined as $\beta_{F P} \mathcal{A}^{*}(\mathbf{S})$. The coupling $\beta_{F P}$ is fixed uniquely by the normalization condition on $\rho$ (sect. 2.4). For large $\beta_{F P}$, the RT runs together with the FP-action (Fig. 2), therefore $\mathcal{A}_{F P}^{*}$ is the perfect action. For intermediate $\beta_{F P}$ values it is not perfect anymore, but - as we shall see - it performs amazingly well. As we discussed in the previous sections, the FP-action can be determined with the help of classical calculations. For $\kappa=2$ it is very short ranged and a relatively simple parametrization describes it well. This parametrized form (given in Table 4) can be easily simulated. Our cluster Monte Carlo program performed very effectively. We would like to urge workers in this field to replace the standard action by the FP-action as a first approximation towards 'perfectness'. These remarks apply to the $C P^{n}$-model also, especially in studies related to topology (sect. 2.2).

In the following, for simplicity, we shall call the 24-parameter action in Table 4 the $\mathrm{FP}$-action keeping in mind possible effects from the errors of this simple parametrization. In the Appendix we calculated the relation of the $\Lambda$-parameter of the FP-action to that of the standard action. The result

$$
\frac{\Lambda_{F P}}{\Lambda_{S T}}=8.17, \quad(\mathrm{~N}=3, \text { couplings from Table } 4) .
$$

can also be written as

$$
\beta_{F P}=\beta_{S T}-0.334+\mathrm{O}\left(1 / \beta_{S T}\right)
$$

Eq. (33) gives a feeling about the meaning of $\beta_{F P}$. At intermediate $\beta$ values we give - for orientation - the infinite volume correlation length $\xi\left(\beta_{F P}\right)$ at a few $\beta_{F P}$ values: $\xi(1.214) \approx 36, \xi(1.08) \approx 18, \xi(0.85) \approx 6$

\subsubsection{Cut-off dependence of the running coupling}

We discussed this problem in the Introduction already. We have chosen the $g(L)=$ $m(L) L=1.0595$ point from ref. [2] since here the observed cut-off effects in the relation $m(2 L) 2 L$ versus $m(L) L$ were large and opposite in sign with respect to perturbation theory (Fig. 1). We simulated the FP-action on a lattice of spatial 
size $L / a=5$ and tuned $\beta_{F P}$ until $m(L) L$ became close to the prescribed value: at $\beta_{F P}=1.0821$ we got $g(L)=1.0578(5)$. Then measuring on a lattice with $L^{\prime} / a=2 L / a=10$ at the same $\beta_{F P}$ value we had $m(2 L) 2 L=1.2611(9)$. In the time direction the lattice was chosen to be at least 6 -times larger than the finite box correlation length $\xi(L)=1 / m(L)$ and distances larger than three times $\xi$ were used in the fitting procedure to obtain the mass gap. At the end we shifted $g(2 L)$ according to the slight difference between the actual (1.0578) and prescribed (1.0595) value for $g(L)$ obtaining $g(2 L)=1.2638(12)$. For this shift we used the universal curve obtained in [2]. The error from this procedure is negligible. We repeated this calculation at $L=10$ also, for $\beta_{F P}=1.214$ with the results: $m(L) L=1.0613(8)$ and $m(2 L) 2 L=1.2664(18)$. After shifting this gives $g(2 L)=1.2635(22)$. In Fig. 3 these two points are compared with the extrapolated prediction $g(2 L)=1.2641(20)$. No cut-off effects can be seen.円

\subsubsection{The two-point function}

We want to compare the spin-spin correlation function of the standard nearest neighbour model with that of the $\mathrm{FP}$-action on coarse lattices and check the extent of violation of rotation symmetry. In this respect one has to understand the following point. The correlation functions do not describe directly physics since they depend on the way the fields are defined. This is reflected, in general, in the scheme dependence of the wave-function renormalization. Here the relation between the fields of the $\mathrm{FP}$-action and the perfect fields of the continuum is more involved. In the case of a free field, as the derivation in section 2.4 (especially eq. (21)) clearly shows, the field of the FP-action is the integral of the perfect field over a square of length $a$. This definition brings an inherent rotation symmetry breaking in the two-point function. Similarly, in the full $\mathrm{O}(3)$ model the shape of the block used in the RG transformation gives a non-rotation invariant definition for the fields in the FP-action. Of course, physical predictions, like the energy values related to the exponential decay of the two-point function, are rotation symmetric.

The effect discussed above is, however, very small. This can be checked analytically in the free field case and it remains so in the $\mathrm{O}(3)$ non-linear $\sigma$-model even on coarse lattices. We simulated the $\mathrm{FP}$-action and the standard action at correlation length $\sim 3\left(\beta_{F P}=0.7\right.$ and $\beta_{S T}=1.18$, respectively $)$. We have calculated the twopoint function on a $24 \times 24$ periodic lattice. This lattice is large enough to avoid rotation symmetry breaking from the box itself (which is a physical infrared effect) up to distances $\sim 9$. In Figs. 8,9 the measured points are connected by piece-wise straight lines to lead the eyes. No symmetry breaking effects can be seen in the two-point function of the FP-action, while the lattice structure shows up clearly when the standard action is simulated.

\footnotetext{
${ }^{5}$ Surprisingly, even for an extremely coarse lattice, $L / a=3$, (at $\left.\beta_{F P}=0.98\right)$ one obtains $g(2 L)=1.2626(11)$, still with no sign of lattice artefacts.
} 


\section{$3 \quad$ RG transformation at finite $\beta$}

The FP-action is the perfect classical action, but we need the perfect quantum action. Since the FP-action seems to have small cut-off effects even at intermediate $\beta$ values, it is a good starting point to find the RT. Assume that at some $\beta_{F P}$ value the cut-off effects in the predictions of the $\mathrm{FP}$-action $\beta_{F P} \mathcal{A}^{*}(\mathbf{S})$ are negligible. Performing a RG step, the size of the cut-off effects for the blocked action will be the same (i.e. negligible), but the correlation length will be a factor of 2 smaller. This is the basic step we have to do in searching for the perfect action. In the following we shall illustrate that it is relatively easy to determine and parametrize the blocked action, and this blocked action remains short ranged if the RG transformation is chosen properly.

\subsection{The RG transformation}

We shall perform one RG step starting with a $\mathrm{FP}$-action (in its parametrical form as given in Table 4) at $\beta_{F P}=1.0$. At this point the correlation length is $\mathrm{O}(10)$. We use the block transformation defined in eq. (3). There is no reason to assume that the optimal value of the parameter $P$ is $\beta_{F P} \cdot 2$ as it is in the case for large $\beta_{F P}$. Actually, we expect that $P_{\text {opt }}$ goes to a constant rather than to zero for small $\beta_{F P}$. Preliminary runs indicated that $\kappa=2.5$ is close to the optimal choice, so we took $P=\beta_{F P} \cdot 2.5$. In this case the range of interaction of the blocked action turned out to be essentially the same as that of the FP-action. For this reason it is sufficient to start with a $10 \times 10$ lattice and block it down to $5 \times 5$. At this small lattice sizes the Ferrenberg-Swendsen [20] technique becomes especially effective and we used it extensively.

\subsection{Determination of the properties of the blocked action}

In a $\mathrm{MC}$ calculation it is relatively easy to determine the change of the action under the change of the configuration. This information will be enough for us. Let us introduce the notation

$$
T(\mathbf{R}, \mathbf{S})=\sum_{n_{B}}\left[P \mathbf{R}_{n_{B}} \sum_{n \in n_{B}} \mathbf{S}_{n}-\ln Y_{N}\left(P\left|\sum_{n \in n_{B}} \mathbf{S}_{n}\right|\right)\right] .
$$

Eq. (3) gives then

$$
\mathrm{e}^{-\beta^{\prime}\left[\mathcal{A}^{\prime}(\mathbf{R})-\mathcal{A}^{\prime}\left(\mathbf{R}_{0}\right)\right]}=\left\langle\mathrm{e}^{T(\mathbf{R}, \mathbf{S})-T\left(\mathbf{R}_{0}, \mathbf{S}\right)}\right\rangle_{\mathbf{R}_{0}}
$$

where

$$
\langle\mathcal{O}\rangle_{\mathbf{R}_{0}}=\frac{\int_{\mathbf{S}} \mathrm{e}^{-\beta_{F P} \mathcal{A}^{*}(\mathbf{S})+T\left(\mathbf{R}_{0}, \mathbf{S}\right)} \mathcal{O}}{\int_{\mathbf{S}} \mathrm{e}^{-\beta_{F P} \mathcal{A}^{*}(\mathbf{S})+T\left(\mathbf{R}_{0}, \mathbf{S}\right)}}
$$




\begin{tabular}{|c|c||c|c|}
\hline$r$ & $\beta^{\prime} \rho^{\prime}(r)$ & $r$ & $\beta^{\prime} \rho^{\prime}(r)$ \\
\hline$(1,0)$ & $0.549(1)$ & $(2,0)$ & $0.0037(14)$ \\
$(1,1)$ & $0.167(1)$ & $(2,1)$ & $0.0027(13)$ \\
& & $(2,2)$ & $0.0002(13)$ \\
\hline
\end{tabular}

Table 5: After one RG step on the FP-action at $\beta=1.0$ the new action $\beta^{\prime} \mathcal{A}^{\prime}$ is parametrized according to eq. (12'). The leading couplings in $\beta^{\prime} \rho^{\prime}(r)$ are given above. The conventions in eq. (40) give $\beta^{\prime}=0.92(2)$.

and $\{\mathbf{R}\}$ and $\left\{\mathbf{R}_{0}\right\}$ are two different block-configurations.

The difference $T(\mathbf{R}, \mathbf{S})-T\left(\mathbf{R}_{0}, \mathbf{S}\right)$ depends on the block-averages

$$
\mathbf{f}_{n_{B}}=\sum_{n \in n_{B}} \mathbf{S}_{n} .
$$

Simulating the effective action defined in eq. (36) with some fixed $\left\{\mathbf{R}_{0}\right\}$ and storing $\left\{\mathbf{f}_{n_{B}}\right\}$ after every sweep, the expectation value in eq. (35) can be calculated for any configuration $\{\mathbf{R}\}$. In practice $\{\mathbf{R}\}$ should be close to $\left\{\mathbf{R}_{0}\right\}$, otherwise the statistical errors can not be controlled.

Specifically, one can chose $\left\{\mathbf{R}_{0}\right\}$ to be the trivial configuration (all the spins point in the $1^{\text {st }}$ direction). In this case $\mathcal{A}^{\prime}\left(\mathbf{R}_{0}\right)=0$ (by definition; the constant generated by the RG transformation does not interest us), and we can calculate $\mathcal{A}^{\prime}(\mathbf{R})$ for configurations which are close to the trivial one. By taking a parametrization for $\mathcal{A}^{\prime}$ in the form of eq. (12') we get

$$
\beta^{\prime} \rho^{\prime}(r)=P^{2} Q^{\perp}(r), \quad r \neq 0,
$$

where $(N=3)$

$$
Q^{\perp}\left(n_{B}^{\prime}-n_{B}\right)=\frac{1}{2}\left\langle f_{n_{B}}^{2} f_{n_{B}^{\prime}}^{2}+f_{n_{B}}^{3} f_{n_{B}^{\prime}}^{3}\right\rangle_{\mathbf{R}_{0}=t r i v} .
$$

The conventions

$$
\sum_{r} \rho^{\prime}(r)=0, \quad \sum_{r} \rho^{\prime}(r) r^{2}=-4
$$

determine then $\rho^{\prime}(0)$ and $\beta^{\prime}$, respectively. We summarized the results in Table 5. It is interesting to observe that the structure of $\rho^{\prime}(r)$ is quite similar to $\rho(r)$. Most importantly, it is similarly short ranged.

We can now take configurations $\{\mathbf{R}\}$, where a single spin is rotated by an angle $\vartheta$, in a trivial background. The corresponding value of $\beta^{\prime} \mathcal{A}^{\prime}$ is given in the second column of Table 6 . As in the case of the $\mathrm{FP}, \mathcal{A}^{*}$, one observes a simple power-like 


\begin{tabular}{|c|c|c||c|c|}
\hline \multirow{2}{*}{$\vartheta$} & \multicolumn{2}{|c|}{ one spin rotated } & \multicolumn{2}{|c|}{ two spins rotated } \\
\cline { 2 - 5 } & $\beta^{\prime} \mathcal{A}^{\prime}$ & $1.432 \vartheta^{2}$ & $\beta^{\prime} \mathcal{A}^{\prime}$ & direct $(2,0)$ interaction \\
\hline 0.09967 & $0.01427(1)$ & 0.01423 & $0.02850(2)$ & $0.000037(28)$ \\
$\pi / 4$ & $0.8780(7)$ & 0.8833 & $1.753(2)$ & $0.0029(23)$ \\
$3 \pi / 8$ & $1.953(3)$ & 1.987 & $3.91(1)$ & $0.000(14)$ \\
$\pi / 2$ & $3.42(1)$ & 3.53 & $6.91(6)$ & $-0.08(6)$ \\
$5 \pi / 8$ & $5.22(3)$ & 5.52 & & \\
$3 \pi / 4$ & $7.26(6)$ & 7.95 & & \\
2.8 & $9.31(9)$ & 11.23 & & \\
3.0 & $9.8(1)$ & 12.89 & & \\
\hline
\end{tabular}

Table 6: One and two spins (at a separation $(2,0)$ ) are rotated in a trivial background. The two-spin result $\left(4^{\text {th }}\right.$ column) is almost exactly twice of the one spin result, indicating that the direct $(2,0)$ interaction is small. The two leading couplings of $\rho^{\prime}$, giving $1.432 \vartheta^{2}$, dominate the action even at large angles.

behaviour in $\vartheta$, which justifies a parametrization in the form of eq. (12'). Actually, the two important couplings in $\rho^{\prime}$ which give a contribution $1.432 \vartheta^{2}$ (third column in Table 6) dominate the results even at large angles $\vartheta$. When two spins, separated by the vector $(2,0)$, are rotated together, the measured action (column 4 in Table 6 ) is very closely twice of the result for a single rotated spin. The direct $(2,0)$ coupling is small.

Since $\mathcal{A}^{\prime}$ is short ranged, for the general parametrization we might consider the type of couplings used for the FP-action before. Actually, as we shall see, the FP-action (as parametrized in Table 4.) $\beta_{F P}^{\prime} \mathcal{A}^{*}$ describes $\beta^{\prime} \mathcal{A}^{\prime}$ quite well with $\beta_{F P}^{\prime} \sim 0.85$. In applications the blocked action $\beta^{\prime} \mathcal{A}^{\prime}$ will be used in a MC simulation, therefore we want a parametrization which gives the value of the action precisely for typical configurations. We generated 300 configurations by $\mathrm{MC}$ using the $\mathrm{FP}-$ action with $\beta_{F P}^{\prime}=0.85$. We calculated the value of the blocked action $\beta^{\prime} \mathcal{A}^{\prime}$ on these configurations using the technique discussed before. The type of the couplings kept for the fit where those used for the FP-action, plus the $(2,0),(2,1)$ and $(2,2)$ two-spin couplings and their powers up to $\vartheta^{6}$. In order to see the quality of the fit and to separate in the fitting error the statistical (coming from $\mathrm{MC}$ determination of the action) and systematical (coming from parametrization) errors we kept those configurations only where the statistical error was below a certain cut value. Fig. 10 shows the error of the fit against the value of the action. The average value of the fit error is 0.004 . If the $(2,0),(2,1)$ and $(2,2)$ couplings are left out, the average fit error is increased to 0.010 . If needed, the quality of the fit can be increased by including further couplings (and increasing the statistical precision). In our feasibility study 
we did not investigate this problem further. We want to mention two additional points only. The $\mathrm{FP}$-action $\beta_{F P}^{\prime} \mathcal{A}^{*}$ with $\beta_{F P}^{\prime}=0.848$ gives a quite reasonable fit also with an average fit-error of 0.022 . The second remark concerns the value $\kappa=2.5$ which makes the blocked action somewhat more local and closer to the FP-action than $\kappa=2$ we used at $\beta=\infty$. At $\kappa=2$, for example, $\beta^{\prime} \rho^{\prime}(2,0)=0.011(1)$, a factor of $\sim 3$ larger than at $\kappa=2.5$. Similarly, the fit-errors (analogous to those discussed above) are increased by a factor of $\sim 2$ if $\kappa=2$ is taken.

Acknowledgement. The authors are indebted for the useful discussions to M. Blatter, R. Burkhalter, G. Mack, K.-H. Mütter, A. Pordt, K. Schilling, P. Weisz, U. Wolff and U. Wiese. We thank M. Lüscher for raising our attention to a minor flaw in the proof of the statement on classical solutions in the preprint version of this paper.

\section{A The Lambda parameter}

The $\Lambda$ parameter for some specific lattice regularizations (in units of $\Lambda_{S T}$ corresponding to the standard action, for example) has been obtained earlier [21, 22]. We give here the result for the generic case, for an action of the form given by eq. (12).

The simplest way to calculate the ratio of the $\Lambda$ parameters is to introduce a chemical potential $h$ [25] coupled to a Noether charge of the $\mathrm{O}(N)$ symmetry and use the fact that the free energy $f(h)$ depends on $h$ in a universal way [25].

Technically, the chemical potential is a constant, imaginary gauge potential: $A_{\mu} \rightarrow i h \delta_{\mu 0} q$, where $q$ is a generator of an $\mathrm{O}(N)$ rotation. We choose a rotation in the 1,2 plane:

$$
q=\left(\begin{array}{cccc}
0 & -i & & 0 \\
i & 0 & & 0 \\
& & \ddots & 0 \\
0 & 0 & & 0
\end{array}\right)
$$

In the presence of the chemical potential the only change in the action is in the scalar product of two spins:

$$
\begin{aligned}
& \mathbf{S}_{n} \mathbf{S}_{n+r} \rightarrow \mathbf{S}_{n} \mathrm{e}^{h r_{0} q} \mathbf{S}_{n+r} \approx \mathbf{S}_{n} \mathbf{S}_{n+r} \\
& \quad+i h r_{0}\left(\mathbf{S}_{n}^{(1)} \mathbf{S}_{n+r}^{(2)}-\mathbf{S}_{n}^{(2)} \mathbf{S}_{n+r}^{(1)}\right)+\frac{1}{2} h^{2} r_{0}^{2}\left(\mathbf{S}_{n}^{(1)} \mathbf{S}_{n+r}^{(1)}+\mathbf{S}_{n}^{(2)} \mathbf{S}_{n+r}^{(2)}\right)+\ldots
\end{aligned}
$$

(In fact, $h$ is here $a h$ and the omitted terms do not contribute in the continuum limit.)

Using the representation in eq. (14) and expanding in $\vec{\pi}$, after straightforward 
calculations we obtain the following expression for the free energy:

$$
\begin{aligned}
& f(h)=-\frac{h^{2}}{2 g}+\frac{1}{2} \int_{q} \ln \left\{\tilde{\rho}(q)+h^{2}+\frac{1}{2} h^{2} \sum_{r} \rho(r) r_{0}^{2} \cos q r-h^{2} \sum_{n_{1}, \ldots, n_{4}} c\left(n_{1}, n_{2} ; n_{3}, n_{4}\right)\right. \\
& \left.\times\left[8 r_{0} r_{0}^{\prime} \cos q d \sin \frac{q r}{2} \sin \frac{q^{\prime} r}{2}+r_{0}^{2}\left(1-\cos q r^{\prime}\right)+r_{0}^{\prime 2}(1-\cos q r)\right]\right\} \\
& +\frac{1}{2}(N-2) \int_{q} \ln \left\{\tilde{\rho}(q)+h^{2}-h^{2} \sum_{n_{1}, \ldots, n_{4}} c(\ldots)\left[r_{0}^{2}\left(1-\cos q r^{\prime}\right)+r_{0}^{\prime 2}(1-\cos q r)\right]\right\} .
\end{aligned}
$$

Expanding in $h$ and using the fact that $f(h)-f(0)$ is the same for any two regularizations, one obtains:

$$
-\frac{1}{g}+Q=-\frac{1}{g^{\prime}}+Q^{\prime}
$$

where

$$
\begin{aligned}
Q= & \int_{q} \frac{1}{\tilde{\rho}(q)}\left\{N-2-\frac{1}{4} \sum_{r} \rho(r) r^{2}(1-\cos q r)\right. \\
& -4 \sum_{n_{1}, \ldots, n_{4}} c\left(n_{1}, n_{2} ; n_{3}, n_{4}\right)\left(r \cdot r^{\prime}\right) \cos q d \sin \frac{q r}{2} \sin \frac{q^{\prime} r}{2} \\
& \left.-\frac{1}{2}(N-1) \sum_{n_{1}, \ldots, n_{4}} c\left(n_{1}, n_{2} ; n_{3}, n_{4}\right)\left[r^{2}\left(1-\cos q r^{\prime}\right)+r^{\prime 2}(1-\cos q r)\right]\right\} .
\end{aligned}
$$

Here we introduced the notations for the relative coordinates in $c\left(n_{1}, n_{2} ; n_{3}, n_{4}\right)$ :

$$
r=n_{1}-n_{2}, \quad r^{\prime}=n_{3}-n_{4}, \quad d=\frac{1}{2}\left(n_{1}+n_{2}-n_{3}-n_{4}\right),
$$

and used the $90^{\circ}$ symmetry to replace e.g. $2 r_{0} r_{0}^{\prime}$ by the scalar product $\left(r \cdot r^{\prime}\right)$.

Eq. (44) gives for the ratio of two $\Lambda$ parameters:

$$
\frac{\Lambda^{\prime}}{\Lambda}=\exp \left\{-\frac{2 \pi}{N-2}\left(Q^{\prime}-Q\right)\right\}
$$

Note that only the 2- and 4-spin interactions contribute when the action is written in the form of eq. (12).

In ref. 22 the $\Lambda$ parameter has been calculated for a particular form of the 4-spin interactions. Unfortunately we do not agree with the result quoted there.

Since in numerical simulations one has to work with a restricted set of couplings, it is more informative to have the $\Lambda$ parameter for that particular set. For the couplings used in our simulations the ratio of this $\Lambda$ parameter to that of the standard lattice action is 8.17, as cited in eq. (32). 
The $\Lambda$-parameter is rather sensitive to the coefficients in the action. We did not attempt to calculate it for the whole set of couplings in $\mathcal{A}^{*}(\mathbf{S})$, although this would be a feasible task. It is remarkable that while $\Lambda_{S T}$ is anomalously small compared to those in a continuum regularization (e.g. $\Lambda_{\overline{M S}} / \Lambda_{S T}=27.21$ ), $\Lambda_{F P}$ is much closer to its continuum counterparts.

\section{References}

[1] Proceedings of the "Lattice 92" Conference, Amsterdam, Nucl. Phys. B (Proc. Suppl.) 30 (1993).

[2] M. Lüscher, P. Weisz and U. Wolff, Nucl. Phys. B359 (1991) 221.

[3] K. Symanzik, in New developments in gauge theories, ed. G. 't Hooft (Plenum, New York, 1980); in Lecture Notes in Physics, 153, ed. R. Schrader et al. (Springer, Berlin, 1982); in Non-perturbative field theory an QCD, ed. R. Jengo et al. (World Scientific, Singapore, 1983),

K. Symanzik, Nucl. Phys. B226 (1983) 187; 205,

B. Berg, I. Montvay and S. Meyer, Nucl. Phys. B235[FS11] (1984) 149,

G. Martinelli, G. Parisi and R. Petronzio, Phys. Lett. 100B (1981) 485,

G. Parisi, Nucl. Phys. B254 (1985) 58,

P. Weisz, Nucl. Phys. B212 (1983) 1,

P. Weisz and R. Wohlert, Nucl. Phys. B236 (1984) 397,

G. Curci, P. Menotti and G. Paffuti, Phys. Lett. 130B (1983) 205,

B. Berg, A. Billoire, S. Meyer and C. Panagiotakopoulos, Comm. Math. Phys. 97 (1985) 31,

M. Falconi et al., Nucl. Phys. B225[FS9] (1983) 313,

M. Lüscher and P. Weisz, Comm. Math. Phys. 97 (1985) 59,

M. Lüscher and P. Weisz, Nucl. Phys. B240[FS12] (1984) 349,

B. Sheikholeslami and R. Wohlert, Nucl. Phys. B259 (1985) 572.

[4] S. H. Shenker and J. Tobochnik, Phys. Rev. B22 (1980) 4462,

A. Hasenfratz and T. Margaritis, Phys. Lett. 133B (1983) 211,

Y. Iwasaki and T. Yoshié, Phys. Lett. B125 (1983) 197, 201; ibid B130 (1983) 77 ,

Y. Iwasaki and S. Sakai, Nucl. Phys. B248 (1984) 441,

Y. Iwasaki, S. Sakai and T. Yoshié, Phys. Lett. 136B (1984) 73,

S. Itoh, Y. Iwasaki and T. Yoshié, Nucl. Phys. B250 (1985) 312,

Y. Iwasaki, Nucl. Phys. B258 (1985) 141,

K.-H. Mütter and K. Schilling, Nucl. Phys. B230[FS10] (1984) 275,

S. Güsken et al., Nucl. Phys. B314 (1989) 63,

Ph. de Forcrand et al. Phys. Lett. B200 (1988) 143,

S. Güsken et al., Phys. Lett. B212 (1988) 216; Nucl. Phys. B327 (1989) 763, 
I. Barbour, E. Laermann, Th. Lippert and K. Schilling, Phys. Rev. D46 (1992) 3618 .

R. Gupta, in Quantum Fields on the Computer, ed. M. Creuz, World Scientific, 1992.

[5] K. Wilson and J. Kogut, Phys. Rep. C12 (1974) 75,

K. Wilson, Rev. Mod. Phys. 47 (1975) 773, ibid 55 (1983) 583.

[6] K. Wilson, in Recent Developments of Gauge Theories, eds. G.'t Hooft et al. (Plenum Press, 1980).

[7] P. Hasenfratz, in "Quarks and leptons and their constituents", (Erice, 1984), ed. A. Zichichi (Plenum, New York, 1988).

[8] A. Migdal, Sovj. Phys. JETP 42 (1975) 413; 42 (1976) 743,

L. Kadanoff, Am. Phys. (NY) 100 (1976) 359; Rev. Mod. Phys. 49 (1977) 267.

[9] For a summary and further references, see ref. [7] above and

R. Gupta, Food for Thought: Five Lectures on Lattice Gauge Theory, Lectures at China Center of Advanced Science and Technology Symposium; Workshop on Lattice Gauge Theory Using Parallel Processors, Gordon and Breach, 1987, P. Hasenfratz and F. Niedermayer, Nucl. Phys. B337 (1990) 233.

[10] U.-J. Wiese, Jülich preprint, HLRZ-41/93 (1993),

T. Balaban, M. O'Carroll and R. Schor, Lett. Math. Phys 17 (1988) 209; Comm. Math. Phys. 122 (1989) 233.

[11] P. Hasenfratz and H. Leutwyler, Nucl. Phys. B343 (1990) 241.

[12] R. H. Swendsen, Monte Carlo Methods, lectures at the Scottish Universities Summer School in Physics (1983),

A. Hasenfratz, P. Hasenfratz, U. Heller and F. Karsch, Phys. Lett. 140B (1984) 76; ibid 143B (1984) 193,

K. C. Bowler et al. Phys. Lett. 163B (1985) 367; Nucl. Phys. B257[FS14] (1985) 155; Phys. Lett. 179B (1986) 375.

[13] Y. Iwasaki and T. Yoshié, Phys. Lett. B131 (1983) 159.

[14] M. Blatter, R. Burkhalter, P. Hasenfratz and F. Niedermayer, work in progress

[15] T. L. Bell and K. Wilson, Phys. Rev. B10 (1974) 3935.

[16] J. E. Hirsch and S. H. Shenker, Phys. Rev. B27 (1983) 1736.

[17] K. Gawedzki and A. Kupiainen, Comm. Math. Phys. 77 (1980) 31

[18] A. Brandt, Math. Comp. 31 (1977) 333. 
[19] R. H. Swendsen and J.-Sh. Wang, Phys. Rev. Lett. 58 (1987) 86, F. Niedermayer, Phys. Rev. Lett. 61 (1988) 2026, U. Wolff, Phys. Rev. Lett. 62 (1989) 361.

[20] A. M. Ferrenberg and R. H. Swendsen, Phys. Rev. Lett. 61 (1988) 2635; ibid 63 (1989) 1195.

[21] G. Parisi, Phys. Lett. 92B (1980) 133, B. Berg, Z. Phys. C20 (1983) 243.

[22] Y. Iwasaki and T. Yoshié, Phys. Lett. B215 (1983) 201, Y. Iwasaki, Nucl. Phys. B258 (1985) 141.

[23] G. Mack, Cargèse lectures, in Nonperturbative Quantum Field Theory, eds. G. 't Hooft et al., Plenum Press, N.Y. 1988, G. Mack and A. Pordt, Comm. Math. Phys. 97 (1985) 267, G. Mack and K. Pinn, Phys. Lett. B173 (1986) 434.

[24] R. H. Swendsen, Phys. Rev. Lett. 52 (1984) 1165, R. Gupta and R. Cordery, Phys. Lett. A105 (1984) 415, R. Gupta, in "Advances in Lattice Gauge Theory", eds. D. W. Duke and J. F. Owens (World Scientific, 1985), G. Murthy and R. Shankar, Phys. Rev. Lett. 54 (1985) 1110.

[25] P. Hasenfratz, M. Maggiore and F. Niedermayer, Phys. Lett. B245 (1990) 522, P. Hasenfratz, F. Niedermayer, Phys. Lett. B245 (1990) 529. 


\section{Figure captions}

Figure 1. The cut-off dependence of $m(2 L) 2 L$ for fixed value of $m(L) L=1.0595$, taken from [2]. The values of $L / a$ are indicated in the plot. The curve is the result of a fit with a second order polynomial in $(a / L)^{2}$. The open box shows the extrapolated value.

Figure 2. Flow of the couplings under RG transformation in the $\mathrm{O}(N)$ non-linear $\sigma-$ model.

Figure 3. The same as Fig. 1 with addition of two points generated by using the $\mathrm{FP}-$ action. These data show no cut-off dependence and agree with the value extrapolated to $a / L=0$ for the standard action.

Figure 4. Dependence of the two-spin interaction coefficient $\rho(r)$ from the distance $r=\left(r_{0}, 0\right)$ for different choices of the block spin transformation. The circles and boxes correspond to the optimal choice with $\kappa=2$, and $\kappa=\infty$, respectively.

Figure 5. The energy spectrum of the lattice regularized free field theory for different lattice actions: the FP (exact) (solid line), the approximation where the two leading couplings of the FP are kept only (dotted line), the standard action(dashed-dotted line) and the Symanzik tree level improved action (dashed line). Note that in the last case the eigenvalue becomes complex above $\left|q_{1}\right| \approx 1.85$. For these values the real part is plotted.

Figure 6 . The value of $\mathcal{A}^{*}(S)$ when one of the spins is rotated by an angle $\vartheta$ with respect to a trivial background of parallel spins. The curve is the contribution from the quadratic and quartic terms in eq. (12'), $\mathcal{A}_{1}(\vartheta)=3.2403\left(\vartheta^{2} / 2\right)-0.0476\left(\vartheta^{2} / 2\right)^{2}$.

Figure 7 . The quality of the parametrization of $\mathcal{A}^{*}$ in Table 1 is shown in this scatter plot. The deviation from the true value of the action is plotted against the value of the action itself for $\approx 500$ configurations.

Figure 8. The spin-spin correlation function as measured with the standard action at $\beta_{S T}=1.18$ corresponding to a correlation length $\sim 3$. The points are connected by piece-wise straight lines.

Figure 9. The same as in Fig. 8 for the $\mathrm{FP}$-action at $\beta_{F P}=0.7$ corresponding to the same correlation length.

Figure 10. The quality of the parametrization for the action $\beta^{\prime} \mathcal{A}^{\prime}$ obtained after a block transformation on the $\mathrm{FP}$-action at $\beta_{F P}=1.0$ is shown here. The fit included the interactions displayed in Table 4 plus the $(2,0),(2,1)$ and $(2,2)$ couplings. 


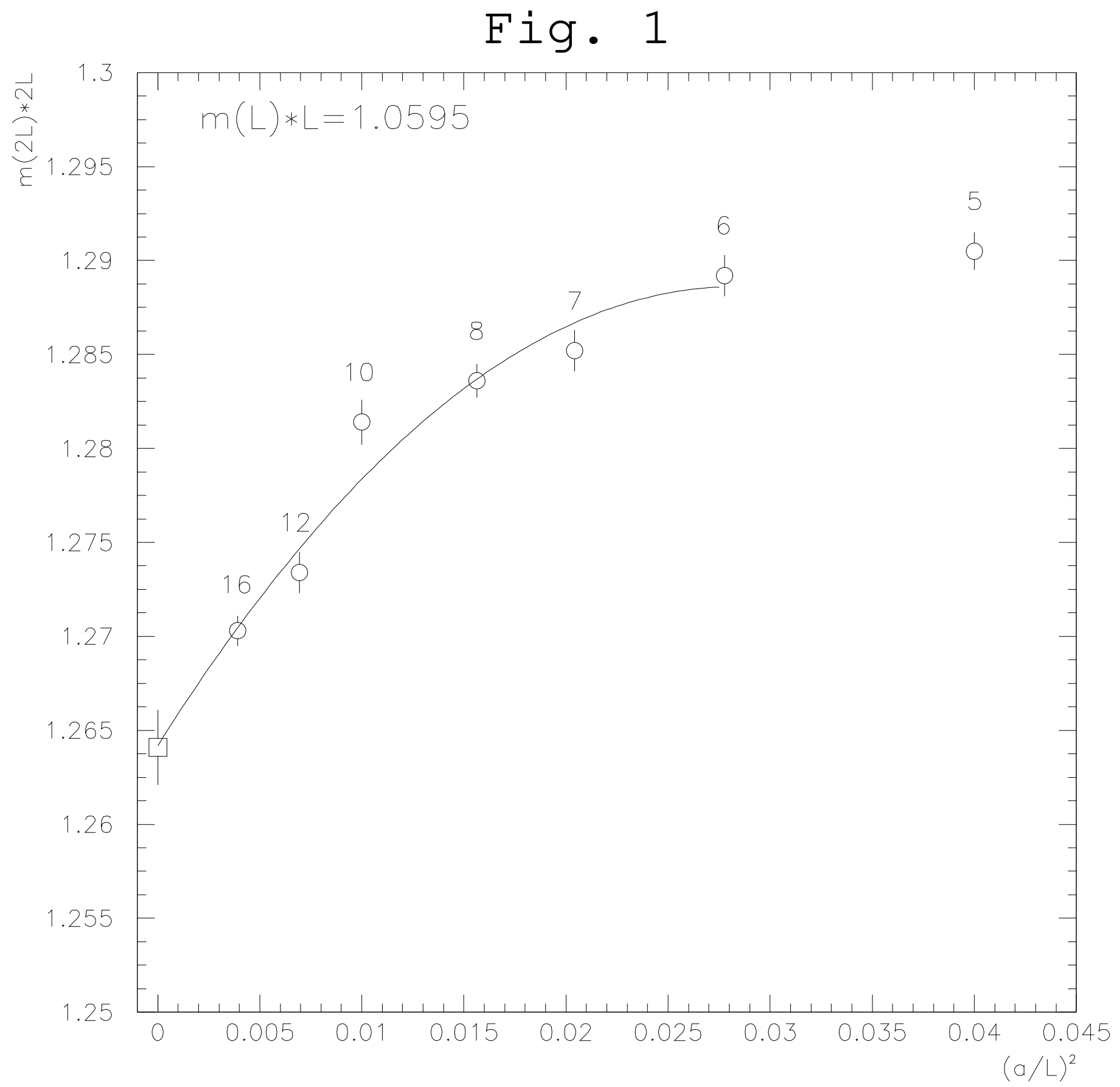




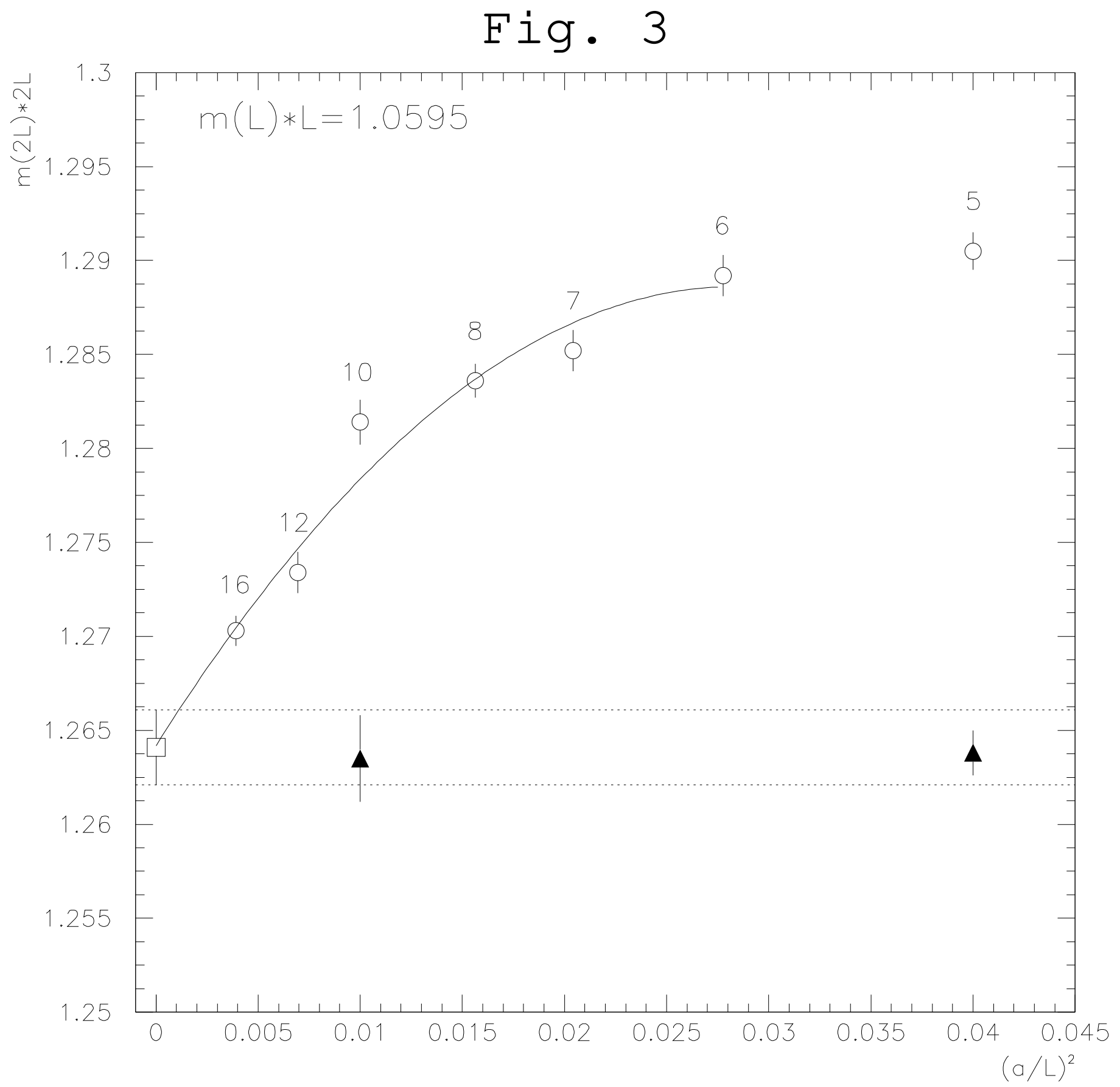




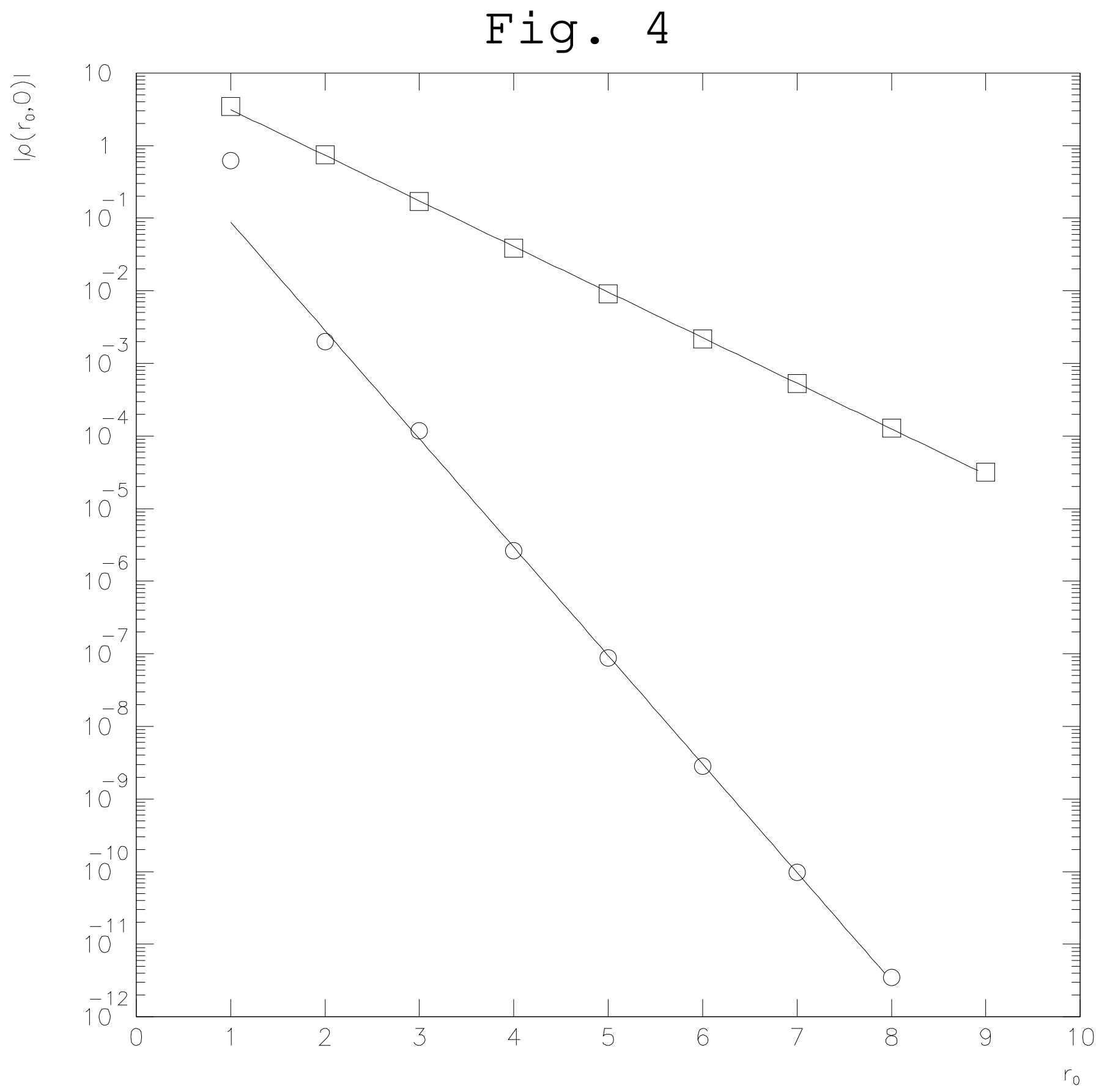




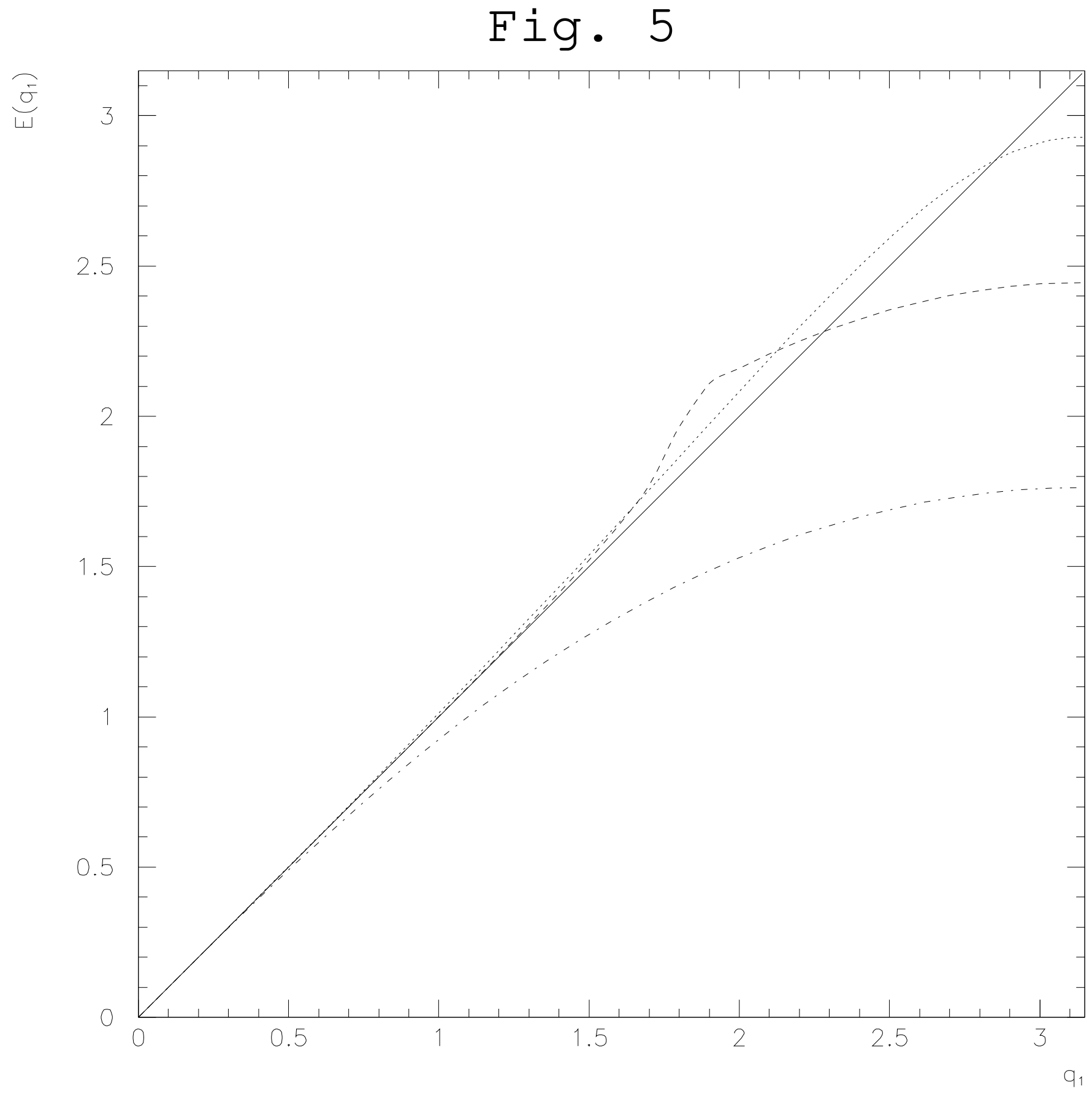


Fig. 6

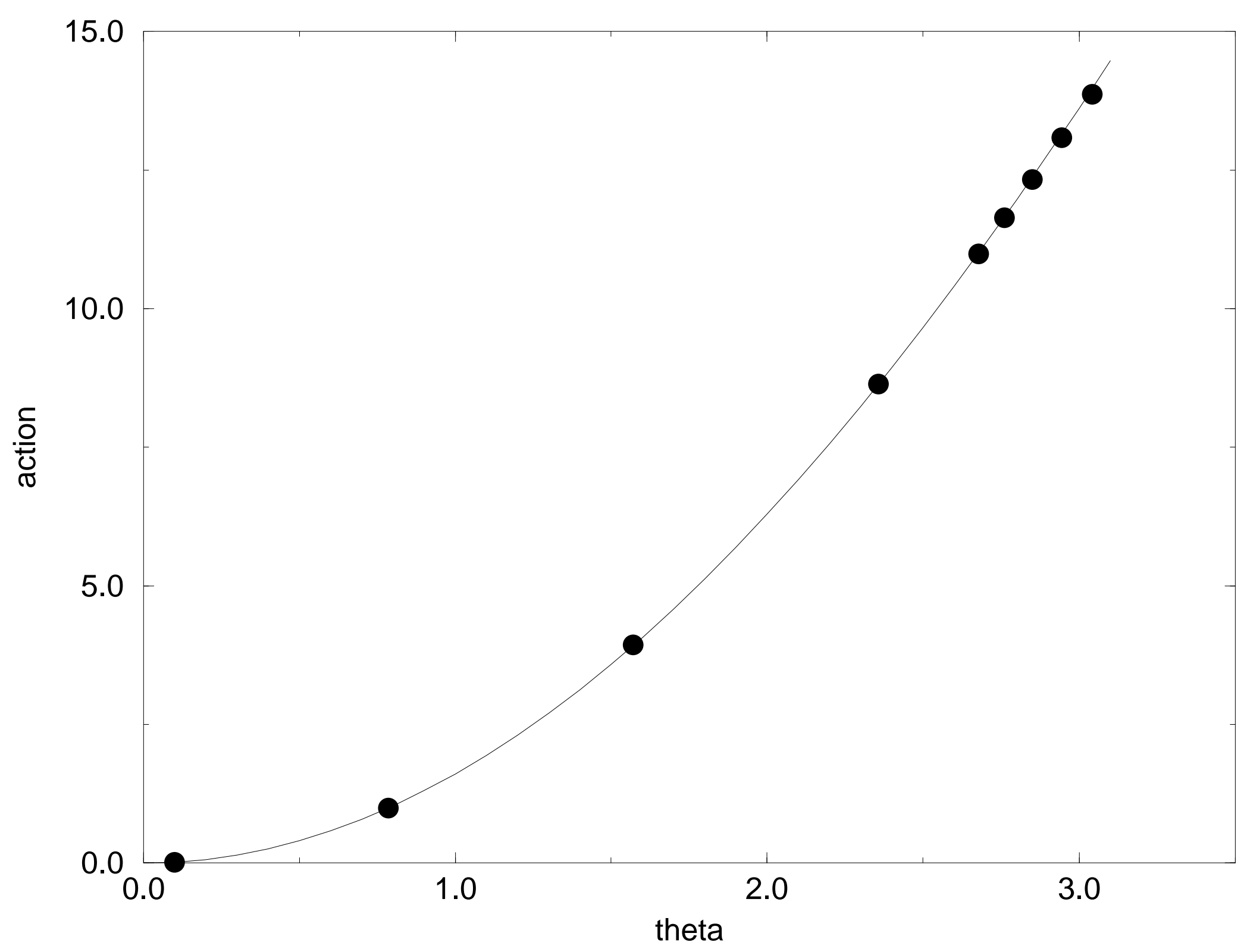


Fig. 7

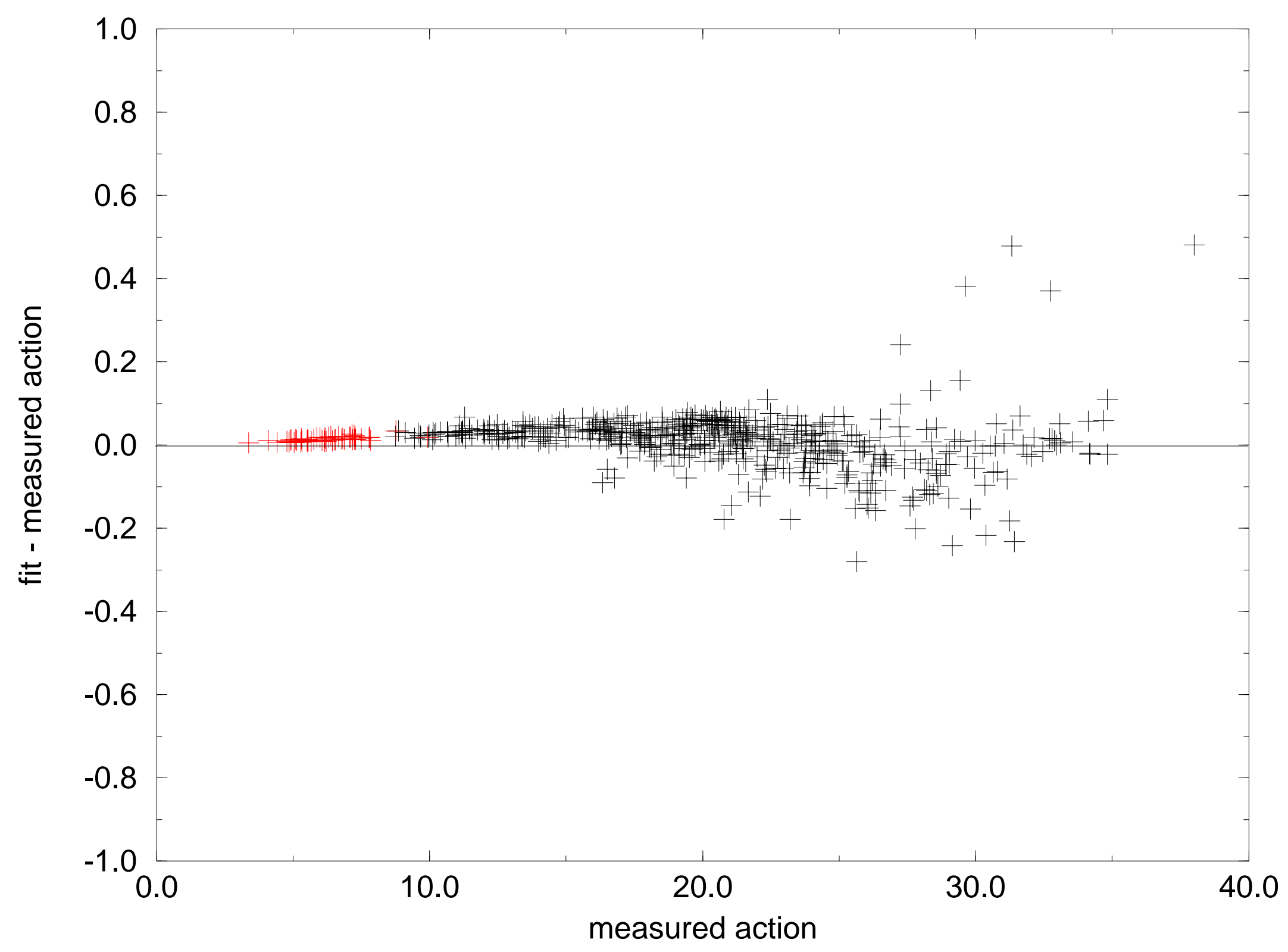


Fig. 8

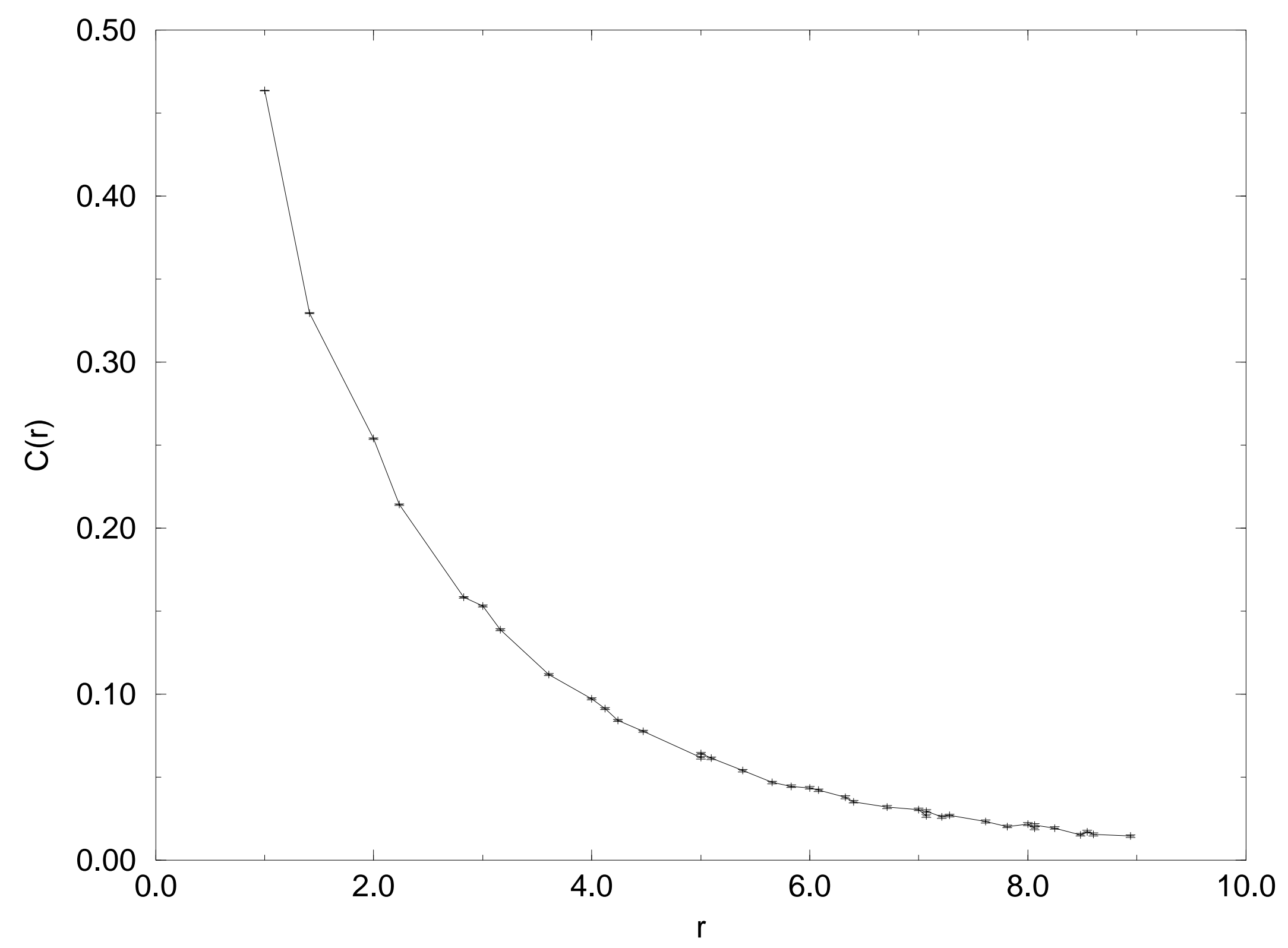


Fig. 9

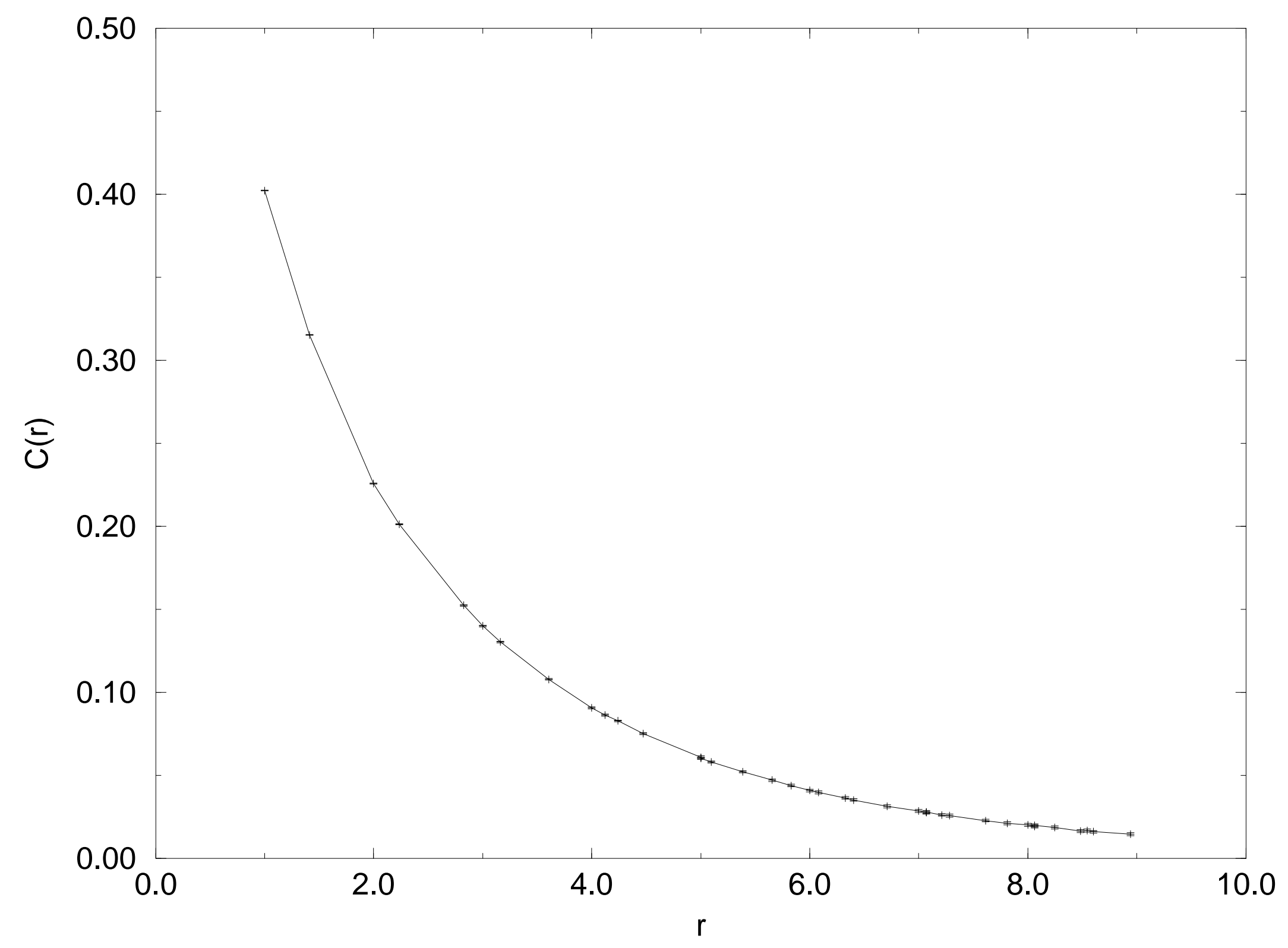


Fig. 10

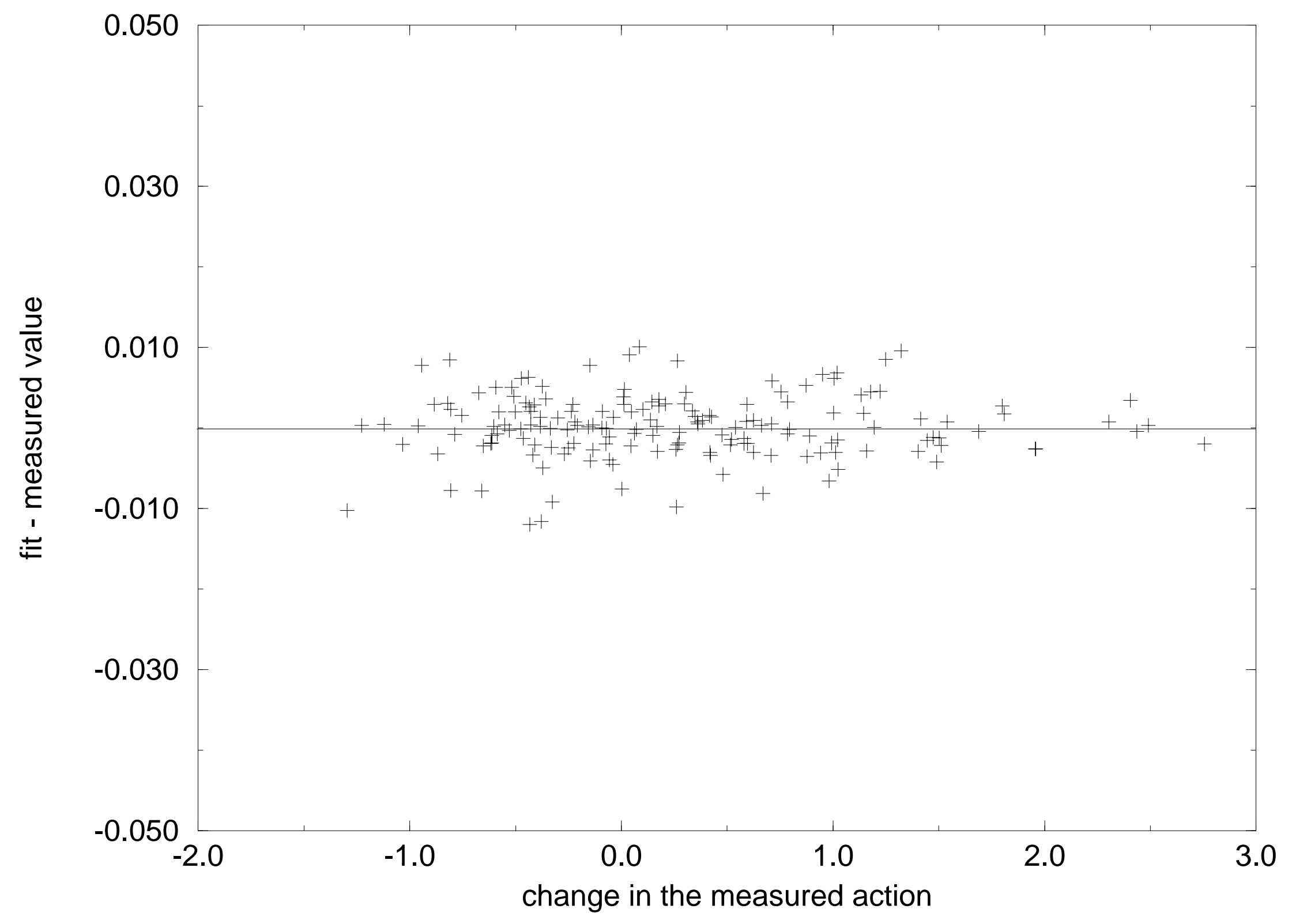

